6-27-2013

\title{
The Effect of Corticision and Different Force Magnitudes on Orthodontic Tooth Movement in a Rat Model
}

Christopher A. Murphy DMD, MDS

University of Connecticut School of Medicine and Dentistry, chrismurphydmd@gmail.com

\section{Recommended Citation}

Murphy, Christopher A. DMD, MDS, "The Effect of Corticision and Different Force Magnitudes on Orthodontic Tooth Movement in a Rat Model" (2013). Master's Theses. 455.

https://opencommons.uconn.edu/gs_theses/455 


\section{The Effect of Corticision and Different Force Magnitudes on \\ Orthodontic Tooth \\ Movement in a Rat Model}

Christopher A. Murphy

B.S. Providence College, 2004

D.M.D. University of Connecticut School of Dental Medicine, 2010

A Thesis Submitted in Partial Fulfillment of the Requirements for the Degree of Master of Dental Science at the University of Connecticut 2013 


\title{
APPROVAL PAGE
}

\author{
Master of Dental Science
}

The Effect of Corticision and Different Force Magnitudes on

Orthodontic Tooth Movement in a Rat Model

Presented by

Christopher A. Murphy, D.M.D.

Major Advisor

Flavio Uribe, D.D.S., M.D.S.

Associate Advisor

Ravindra Nanda, B.D.S., M.D.S., Ph.D.

Associate Advisor

Ivo Kalajzic, M.D., Ph.D.

Associate Advisor

Jianjun Hao, D.D.S., Ph.D.

University of Connecticut

2013 


\section{TABLE OF CONTENTS}

$\underline{\text { Title }} \quad \underline{\text { Page }}$

$\begin{array}{ll}\text { Title Page } & 1\end{array}$

$\begin{array}{ll}\text { Approval Page } & 2\end{array}$

Table of Contents $\quad 3$

$\begin{array}{ll}\text { Abstract } & 4\end{array}$

$\begin{array}{ll}\text { Chapter I - Introduction } & 7\end{array}$

$\begin{array}{ll}\text { Background } & 7\end{array}$

Anatomy, Biological Responses and Orthodontic Tooth Movement 7

$\begin{array}{ll}\text { Tooth Movement Models } & 13\end{array}$

$\begin{array}{ll}\text { Rationale and Objectives } & 19\end{array}$

$\begin{array}{ll}\text { Chapter II - Hypothesis and Aims } & 20\end{array}$

Hypothesis and General Objectives $\quad 20$

Specific Aims and Objectives 20

$\begin{array}{ll}\text { Chapter III - Materials and Methods } & 21\end{array}$

$\begin{array}{ll}\text { Experimental Animals } & 21\end{array}$

$\begin{array}{ll}\text { Experimental Design } & 21\end{array}$

Method of Force Application 22

Application of Corticision $\quad 24$

Measurement of Tooth Movement and Micro CT Analysis 24

Dissection and Tissue Preparation $\quad 25$

$\begin{array}{ll}\text { Immunohistochemistry } & 26\end{array}$

$\begin{array}{ll}\text { Statistics } & 27\end{array}$

$\begin{array}{ll}\text { Chapter IV - Results } & 28\end{array}$

Effect of Corticsion and Force Magnitude on Tooth Movement 28

Effect of Corticision and Force Magnitude on the Alveolus 31

Correlation between BVF and Orthodontic Tooth Movement 32 


\begin{abstract}
Background: The rate of orthodontic tooth movement may be influenced by a number of factors including, but not limited to, the magnitude of the applied force and the induction of a regional acceleratory phenomenon resulting from surgical manipulation of the alveolus. The aim of this study was to evaluate the effect of two distinct magnitudes of applied force with and without corticision on the rate of tooth movement and the alveolar response in a rat model.
\end{abstract}

Method: Sixty six week old male rats were divided equally into four groups. Groups included: light force, light force with corticision, heavy force and heavy force with corticision. Force was delivered from the maxillary left $1^{\text {st }}$ molar to the maxillary incisors using prefabricated $10 \mathrm{~g}$ or $100 \mathrm{~g}$ Sentalloy springs. Corticision was done at time of appliance placement and repeated one week afterwards on the mesiopalatal aspect of the left maxillary first molar. The right hemi maxillae served as unloaded controls. Micro computed tomography $(\mu \mathrm{CT})$ was used to evaluate tooth movement and the alveolar response between maxillary $1^{\text {st }}$ and $2^{\text {nd }}$ molars on day 14 . Tartrate resistant acid phosphatase (TRAP) staining of paraffin embedded sections was done to quantify osteoclasts and odontoclasts present. The expression of receptor activator of nuclear factor kappa $ß$ ligand (RANKL) was studied using immunohistochemistry. Histologic sections were used to study the amount of root resorption present.

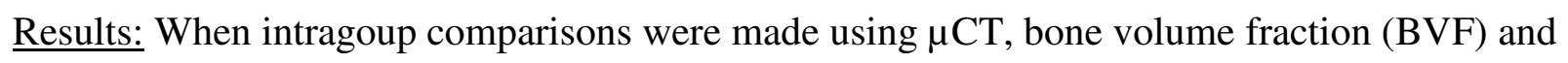
tissue density were found to be significantly less on the loaded sides, with the exception of BVF in the light force group. When intergroup comparisons were made tooth movement, bone volume fraction, apparent density, tissue density, total volume or bone volume showed no significant differences. Histologic analysis found BVF to be decreased in the light force group TRAP staining demonstrated no significant differences in the number of osteoclasts/odontoclasts or 
osteoclast/odontoclast surface between groups. Differences in RANKL expression between groups was found to be non-significant. Corticison had a protective effect against root resorption.

Conclusions: There were no differences in tooth movement or alveolar response observed with $\mu \mathrm{CT}$. Histologic analysis of the furcation area showed light force significantly decreased BVF. Root resorption decreased in animals receiving corticision. 


\section{Chapter I: Introduction}

\section{A. Background}

\section{Anatomy, Biological Responses and Orthodontic Tooth Movement}

Comprehensive orthodontic treatment is a gradual process. Although treatment times vary, the American Association of Orthodontists has estimated that most comprehensive treatment times last between 18 and 30 months (1). Length of therapy is influenced by a number of factors including complexity of the case, skill of the practicing orthodontist, degree of patient cooperation and the rate of tooth movement. Extended treatment times have been associated with negative outcomes such as increased caries (2), periodontal disease $(3,4)$, root resorption (5) and pulpal reactions (6). Decreasing the length of orthodontic therapy by increasing the rate of tooth movement has the benefit of reducing the risk of these unwanted side effects and increasing patient satisfaction.

Orthodontic tooth movement is a dynamic process of alveolar bone remodeling which couples bone resorption and apposition in response to the application of mechanical forces (7). The strains generated by orthodontic forces are transmitted to a tooth's periodontal ligament and supporting alveolar bone producing areas of tension and pressure within the PDL. Changes in the vascularity of the PDL $(8,9)$ and the subsequent release of a number of important factors such as neuropeptides $(10,11)$, cytokines $(10,12,13)$, growth factors $(13)$, colony stimulating factors (13) and arachidonic acid metabolites $(14,15,16)$ promotes a series of complex interactions among dental and paradental cellular populations generating changes in gene expression. Ultimately, these changes in gene expression results in the attraction of leukocytes, osteoclasts and osteoblasts. The migration and activation of these cells creates localized areas conducive for alveolar remodeling with bone resorption occurring in sites of compression and 
bone formation occurring in regions of tension (7). Manipulating factors that affect the rate of resorption and deposition of alveolar bone holds the promise of enhancing tooth movement and reducing treatment times.

The velocity of tooth movement is regulated by bone turnover, bone density and the degree of hyalinization of the PDL in response to the forces being applied. Efforts to enhance the rate of orthodontic tooth movement have targeted these factors and can be categorized as being either pharmacologic or physical. Pharmacologic agents have been shown to influence orthodontic tooth movement by altering bone metabolism. Administration of synthetic analogues of eicosanoids $(17,18,19)$, chronic treatment with corticosteroids $(20,21)$, exogenous applications of parathyroid hormone $(22,23)$, thyroxine $(24,25)$ and 1,25 - dihydroxycaholecalciferol $(19,26$, 27) have been found to increase the velocity of tooth movement. Despite accelerating tooth movement these treatments have also been found to produce a number of negative local and systemic side effects such as hyperalgesia (28), bone loss and osteoporosis, delayed wound healing and root resorption $(29,30)$ which has limited their clinical applications. As a result research has shifted towards physical methods aimed at enhancing tooth movement.

The duration and magnitude of force applied to teeth influence the velocity of tooth movement. Orthodontic force duration can be classified as being continuous, interrupted or intermittent based on the rate of decay. Continuous forces are widely believed to be the most effective means of producing efficient tooth movement $(31,32)$. Clinical experience has revealed that a threshold of 4-8 hours of force application is needed for successful tooth movement with better results found when the length of force duration is increased (33). Research supports this concept of a time threshold, and it has been shown that it takes approximately 3 to 4 hours of maintained force before there are significant elevations of cyclic 
nucleotides in the PDL and alveolar bone acting as second messengers for bone remodeling (34). The original concept of an optimal force for tooth movement was proposed in 1932 by Martin Schwarz. He defined an optimal force as "the force leading to a change in tissue pressure that approximated the capillary vessel's blood pressure, thus preventing their occlusion in the compressed periodontal ligament" (36). Subsequent histological studies would maintain that the optimal force should be kept as light as possible and distributed evenly along the root's surface to minimize contact between the teeth and alveolus, PDL hyalinization and necrosis, undermining resorption and irreversible root resorption $(7,37,38)$. Storey and Smith's research in the 1950's investigating canine retraction would give rise to the belief that there is "an optimum range of force values (150-200 g) that should be used to produce a maximum rate of tooth movement" (39). Forces below this range would produce little if any canine retraction and excessive forces would result in decreased movements. Storey and Smith's findings helped lead to Begg's differential force theory. Begg's theory held that by applying the optimal force needed to move a specific tooth it would be possible to promote that tooth's movement while eliminating unwanted reciprocal movements of anchor segments. More specifically, Begg believed that when light retractive forces (approximately 100 to $150 \mathrm{~g}$ ) were placed on canines, these teeth would retract with no movement of the premolar and molar anchor segment, and when heavy protractive forces (approximately 400 to $500 \mathrm{~g}$ ) were placed on the premolars and molars they would protract with the canine acting as a stable anchor (39). Unsuccessful attempts to replicate the findings of Storey and Smith and contradictory reports have since called the theory of differential forces into question $(40,41,42,43,44)$ and the most efficient means of moving teeth remains controversial. It now appears that the "optimal force" is highly variable among patients and can be best described as the lightest force capable of producing maximal 
tooth movement through frontal resorption (where osteoclasts line up in the margin of alveolar bone adjacent to the compressed PDL, producing direct bone resorption) with minimal patient discomfort $(7,35)$.

A number of alternative physical methods to increase tooth movement have also been studied. These have included the use of pulsed and static magnetic fields $(45,46)$, electrical currents $(47,48)$, lasers (49) and surgical manipulation of the alveolar bone. Surgical methods to enhance the rate of tooth movement have garnered considerable attention in recent years. These methods include alveolar surgery to undermine interseptal bone $(52)$, corticotomies $(53,54,55$, $56,57,58,59)$, dentoalveolar distraction $(60,61,62)$, dental distraction $(60,63,64)$, and corticision $(65,66,67)$. By reducing alveolar resistance to tooth movement and accelerating the turnover rate of the alveolar bone by means of a regional acceleratory phenomenon, these surgical methods have shown substantial increases in the rate of orthodontic tooth movement and reduction of treatment times.

Surgical manipulation of the alveolus to enhance the speed of orthodontic tooth movement dates back to the late $19^{\text {th }}$ century. The use of corticotomy to correct malocclusion was first introduced by Cunningham who in 1893 at the Chicago Dental Congress described "Luxation or Immediate Method in Treatment of Irregular Teeth". He proposed making linear cuts in the cortical plates surrounding teeth as a means of mobilizing teeth for immediate movement (68). Sixty seven years later Heinrich Kole reintroduced the concept. Kole combined interradicular corticotomies and supra apical osteotomies to accelerate tooth movements. He believed that by reducing resistance exerted by the cortical bone of the alveolus it was possible to promote en bloc movement of the entire alveolar cortical segment with the only resistance being provided by the less dense medullary bone (69). Despite demonstrating significant reductions in the time 
needed for active tooth movements, the invasive nature of Kole's technique prevented it from becoming widely accepted. Subsequent publications released by Duker in 1975 (70), Generson et al in 1978 (71), Anholm et al. in 1986 (72), Gantes et al. in 1990 (73) and Suya in 1991 (74) continued to build upon and modify Kole's method (69) demonstrating similar increases in the rate of tooth movement. In 2001 Wilcko and Wilcko developed a revised corticotomy facilitated technique they dubbed accelerated osteogenic orthodontics (AOO) (58) and later changed to periodontally accelerated osteogenic orthodontics (PAOO) (54). Wilcko and Wilcko combined the modified corticotomy facilitated approach proposed by Suya (74) with periodontal therapy including alveolar bone augmentation (58). Unlike Kole, they attributed the rapid rate of tooth movement they observed to a regional acceleratory phenomenon (RAP) rather than bony bloc movement (58).

The regional acceleratory phenomenon was first described by orthopedist Harold Frost in 1983 (75). Frost recognized that any noxious stimulus of sufficient magnitude was capable of inducing an acceleration and domination of most ongoing normal and vital tissue processes in both soft and hard tissues as part of the body's healing process. When the insult was directed at osseous tissue, the result was accelerated bone turnover and reduction in regional bone density leading to a transient state of osteopenia. Subsequent research has confirmed that the acceleration of tooth movement associated with surgical intervention is the result of increased localized bone turnover and osteopenia based on a regional acceleratory phenomenon $(94,95)$.

Although the regional acceleratory phenomenon induced by surgical intervention has demonstrated remarkable increases in the rate of tooth movement, the clinical application of these surgical interventions has been hampered by a number of factors including the duration of the effect and poor patient acceptance due to the invasive nature of these procedures. In a paper 
published by Buschang in 2010 comparing two surgical interventions, dentoalveolar distraction and periodontal distraction, it was found that increased rate and amount of tooth movement was related to the severity of the surgical insult (60). This is consistent with Frost's original observations "that the size of the affected region and the intensity of its response varies directly with the magnitude of that stimulus" (75). Furthermore, it has been shown in multiple studies that the RAP effect on alveolar bone is transient, lasting only 2 to 4 weeks. In an effort to develop a minimally invasive approach to induce a RAP effect without flap reflection that could be repeated with minimal patient discomfort, Kim and Park introduced corticision. With this technique a "reinforced scalpel is used as a thin chisel to separate the interproximal cortices transmucosally without reflecting a flap" (65). In their work using a feline model to investigate the effects of corticision they found in the animals receiving corticision there was extensive bundle bone with less hyalinization and more rapid removal of hyalinized tissue compared to controls. These findings demonstrated corticision to be an efficient means of stimulating tooth movement by inducing a RAP and accelerating bone remodeling.

Compared to the drug- induced approach, a major benefit of surgical assisted orthodontics is that the main effects of RAP seem to be restricted to the site of the stimuli, though areas of close proximity may also be affected. It is hypothesized that the targeted application of corticision coupled with the use of sufficient forces can promote efficient tooth movement. The purpose of this study is to assess the rate of tooth movement and biologic effects of corticision with two distinct force magnitudes on the remodeling of alveolar bone during orthodontic tooth movement in a rat model. 


\section{Tooth Movement Models}

The relationship between the magnitude of orthodontic force and subsequent tooth movement remains controversial with conflicting reports and multiple hypotheses. Complicating the interpretation of published data is the ambiguity of what constitutes a light or heavy force, the nature of tooth movement being studied, differences in time courses being observed, variation in stresses and strains being evoked dependent upon a tooth's root surface area and large variations in biologic responses both between and within the subjects being examined. As a result some authors have concluded that the dentition's response to force is dose dependent with some claiming heavy forces to be most effective $(42,44,83,84)$ and others claiming light forces to be more advantageous $(85,86)$. Still others have claimed there is no association at all between force magnitude, type of tooth movement and the amount of displacement $(87,88,91)$.

Quinn and Yoshikawa (93) suggested four hypotheses to illustrate the possible relationships between force magnitude and tooth movement. With regards to these four hypotheses, experimental data provide the most support for the fourth model which suggests that the relationship between the amount of applied force and tooth movement is linear up to a point, after which additional stress causes no noticeable increase in the rate of tooth movement. The linear portion of the curve is supported by multiple studies that have demonstrated that an increase in force results in increased rates of movement, while the plateau in tooth movement observed at higher force levels could explain the findings of Melsen (87), Pilon (88) and Owman-Moll (91) who found no association between force magnitude and tooth movement. Studies, like those of Storey and Smith (39), Andreason and Zanzinger (44) and van Leeuwen et al (90) demonstrating greater displacement of posterior segments as force levels are increased with minimal anterior movement provide further support for this hypothesis when differences in 
root surface area and distribution of stress levels within the PDL are accounted for. Considering that stresses and strains produced by forces vary inversely with the surface area of the roots, a relatively light force could cause a maximal biologic response anteriorly over a small root surface area, with little effect posteriorly. Meanwhile a much heavier force placed on posterior teeth and dispersed over a much larger root surface area may place those teeth on the linear, ascending portion of the curve and result in greater movement with the anterior segment showing little change having already hit a plateau. In addition, studies that have examined multiple increments of force tend to show that the initial dose response relationship plateaus at higher levels. Burstone and Groves examined the relationship between force magnitude and tooth movement using forces ranging from 25 to $150 \mathrm{~g}$ to retract protrusive anterior teeth and found that increasing the magnitude of force above 50-75 $\mathrm{g}$ did not increase the rate of movement (92). A study conducted by Boester and Johnson revealed a similar plateau when higher forces levels were used to retract canines. They observed that forces above $140 \mathrm{~g}$ produced no measurable increase in tooth movement (43). King et al. also found that the "orthodontic appliance can be overloaded, resulting in no further increases in tooth movement" somewhere between 20 and 40 $\mathrm{g}$ while analyzing molar mesialization in rats $(80)$.

The current body of evidence seems to suggest that at lower levels, forces elicit a dosedependent response. However, at higher levels it appears that the rate of tooth movement is not influenced by force magnitude and has reached its maximal rate dictated by the alveolus' biologic capacity to undergo remodeling. The regional acceleratory phenomenon elicited by surgical manipulation of the alveolus has demonstrated remarkable increases in the rates of tooth movement by accelerating localized alveolar bone turnover (54-75). Kim and Park have demonstrated that even minimally invasive insults to the alveolus by means of corticision can 
invoke a RAP effect and stimulate orthodontic tooth movement $(65,66)$. In their study investigating the effects of corticision on alveolar remodeling they found that cats treated with corticision and a $100 \mathrm{~g}$ retractive force on the canine had increased catabolic remodeling in the direction of tooth movement (65). This was represented by direct bone resorption with less hyalinization and more rapid removal of hyalinized tissue compared to the control group. They also found that corticision enhanced anabolic remodeling as well with 3.5 times more mean appositional bone in the corticision group. Preliminary data from a study conducted at the University of Connecticut corroborate Kim and Park's findings. In this study Vaziri et al. evaluated the protraction of maxillary molars in a rat model over a 14 day period using a 60 gram Niti coil spring with corticision being applied on the mesiopalatal aspect of the maxillary left first molar either at the time of appliance placement, or at the time of appliance placement and one week afterwards. Molar protraction was quantified using feeler gauge measurements taken between the first and second left maxillary molars. It was observed that rats subjected to corticision at the beginning of the experiment and one week after the application of force demonstrated accelerated molar mesialization compared to control animals.

In an effort to understand the nature of the biologic response that facilitates orthodontic tooth movement a large number of experimental studies have been performed on animals such as primates, dogs, cats, rats and mice. Among these species, rats have become the most commonly used animal model accounting for 57 percent of animal studies on orthodontic tooth movement between 1981 and 2002 (76).

Rats have become the favored experimental model to study orthodontic tooth movement for a number of reasons. First, rats are relatively inexpensive and can be housed for long periods of time, making large sample sizes possible. Furthermore, the preparation of rat specimens for 
histologic study is much easier in comparison to other animal models and most antibodies required for cellular and molecular biological techniques are readily and oftentimes only available for rats and mice. In addition, the use of rats opposed to other small rodents is more practical as the placement of orthodontic appliance is not as difficult due to their larger size (76). A major limitation to the use of any animal model relates to how applicable the findings are when compared to humans, and what meaningful conclusions can be drawn from animal experiments. Differences between species therefore must be taken into consideration. It is well known that morphological and physiological differences exist between rats and humans. Obviously, human teeth are much larger than rat teeth with rat molars being approximately 20 times smaller than human molars (77). This size discrepancy must be accounted for as the effect of an applied force is related to the size of the tooth involved and its root surface area (78). In contrast to humans the alveolar bone of rats is denser, lacks osteons and marrow spaces and has little osteoid tissue along the alveolar bone surface (79). These characteristics may influence the processes of bone deposition and resorption. There also exist structural differences in the rat PDL and supporting structures (79) and it has been found that rat root formation and resorption occurs more quickly (79). In addition, rat incisors erupt continuously and rat molars naturally drift distally. These physiologic processes if not considered can lead to inaccurate data (76). Despite these shortcomings, the multiple advantages that come from working with rats make them a good experimental animal model.

In 2004 Ren conducted a systematic review of the use of rats as a model for experimental tooth movement from 1981 to 2002 . The aim of the study was to give a critical evaluation of the use of elastics in this type of research and to propose a well defined experimental model for tooth movement in rats. Inclusion criteria for a good model were a force magnitude of less than $20 \mathrm{cN}$, 
molar mesialization, an experimental duration longer than 2 weeks, and no extra experimental condition such as drug intervention. Ren found that although rats made up 57\% of the animal studies between 1981 and 2002 almost all of these studies failed to meet the inclusion criteria. The majority of the investigations used poorly designed and documented experimental set ups that cast doubt on the value of the data reported regarding the relationship between force and tooth movement. Shortcomings commonly identified included: a failure to account for distal drift of molars and/or continuous eruption of incisors; poor appliance designs that did not account for the decreased rat root surface; force systems using elastics and a variety of coil springs that were not measured at all, were too high or were only measured at the beginning of the experiment; failure to account for force decay and short experimental period (76).

Of the 153 studies examined only three met all of Ren's inclusion criteria for a good experimental model $(80,81,82)$. The three studies were conducted by the same research group and used the same animal model. Adult male Sprague Dawley rats were anesthetized and had modified cleats bonded to the occlusal surface of prepared maxillary first molars. Nine mm lengths of closed coil orthodontic springs (Unitek Hi-T; 0.006 inch; 0.022 inch arbor diameter) were attached to steel ligature wire loops and placed over the molar cleat. The anterior end of the coil was then attached to a premeasured weight and allowed to hang freely until the prescribed amount of force was achieved (20g, 40g or $60 \mathrm{~g})$. Excess coil was trimmed, and the free end of the coil was bonded to the lateral surface of the maxillary incisor. Mandibular first and second molar were extracted to eliminate extraneous forces from occlusion. The animals were divided into 6 time courses extending up to 2 weeks. Tooth movement was quantified from enlarged cephalograms by measuring the position of a reproducible landmark on the molar cleat 
with respect to either zygomatic amalgam implants or a barbed broach placed submucosally on the palate (80).

Although this model represented an improvement over other designs, there were a number of shortcomings. First, only the initial force was controlled and there was no reactivation. Secondly, although $20 \mathrm{cN}$ was used as the lowest force, the medium $(40 \mathrm{cN})$ and high $(60 \mathrm{cN})$ forces applied were quite high. If a human molar is assumed to be 20 times larger than a rat molar, the medium and high forces would have been equivalent to $800 \mathrm{cN}$ and $1200 \mathrm{cN}$, respectively. Third, there were a number of findings indicating poor animal welfare. For instance first and second mandibular molars were extracted, the animals demonstrated a tendency to lose weight during the experiment and the success rate of the appliance was only 79\%. Fourth, the experimental period only lasted 2 weeks. Lastly, continuous incisor eruption was not taken into consideration with the appliance design (76).

In response to these weaknesses Ren developed a new rat model. A split mouth design was chosen in order to account for physiologic distal drift of molars, the physiologic growth of the snout and forward movement of the incisors, continuous eruption of the incisors and potential distal tipping of the incisors used as anchorage. The experimental side was chosen randomly and the contralateral side served as the control. Stainless steel ligature wires $(0.2 \mathrm{~mm}$ in diameter $)$ were bent to connect all three maxillary molars as a unit and a wire Sentalloy® closed coil spring $(10 \mathrm{cN}, 0.22 \mathrm{~mm}$ wire diameter, $0.56 \mathrm{~mm}$ eyelet diameter) was attached to deliver a reproducible force of $10 \pm 2 \mathrm{cN}$ over a range of 3-15 mm activation. The animals were placed under general anesthesia and a transverse hole drilled through the alveolar bone and both maxillary incisors at the mid-root level to accommodate a stainless steel ligature. Upon placement, bonding and activation of the preformed appliance it was attached to the ligature wire through the snout and 
incisors. Intraoral measurements and radiographs were taken at $0,1,2,3,4,8$, and 12 weeks. Intraoral measurements were made from the most mesial point of the maxillary molar unit and CEJ of the ipsilateral maxillary incisor at the gingival level (76).

After giving thorough consideration to the advantages and disadvantages of different animal models used to study orthodontic tooth movement, a rat model was chosen for this experiment. The experimental design selected for the study of orthodontic tooth movement in rats was based upon the work of Ren to identify and address shortcomings encountered in previous rat models (76).

\section{B. Rationale and Objectives}

Orthodontic tooth movement is a dynamic process of coupled bone resorption and apposition in response to prolonged mechanical forces transmitted from the tooth to the PDL and supporting alveolar bone. The rate of orthodontic tooth movement is influenced by a number of factors including, but not limited to, the magnitude of the applied force and the induction of a regional acceleratory phenomenon resulting from surgical manipulation of the alveolus. Kim et al. recently proposed a new less invasive method of stimulating a regional acceleratory phenomenon and accelerating tooth movement in a feline model known as corticision (65). The purpose of this study is to assess the effect of corticision and two distinct force levels on the rate of orthodontic tooth movement and to quantify alveolar changes and localize the osteoclasts and osteoclastogenesis induction molecules (RANKL) responsible for the biological response and resulting tooth movement. Additionally, it is the intention of this study to evaluate the effect of these two distinct force levels and corticision on the severity of root resorption. 


\section{Chapter II: Hypotheses and Aims}

\section{A. Hypotheses and General Objectives}

Hypothesis 1: We hypothesize that the rate of orthodontic tooth movement will be increased in the experimental group with corticision and a heavy protractive force.

Hypothesis 2: We hypothesize that there will be more osteoclasts, higher RANKL expression, decreased bone and tissue density in the experimental group with corticision and a heavy protractive force.

Hypothesis 3: We hypothesize that there will be increased root resorption in the non corticision groups

Null Hypothesis 1: There will be no difference in the amount of tooth movement among the experimental groups with corticision and different applied forces versus the control group.

Null Hypothesis 2: There will be no difference in osteoclast quantity, RANKL expression, bone and tissue density among the experimental groups.

Null Hypothesis 3: There will be no difference in root resorption among the experimental groups

\section{B. Specific Aims and Objectives}

Aim 1: To determine the effect of corticision and two distinct magnitudes of applied force on the amount of orthodontic tooth movement. 
Aim 2: To quantify changes in the alveolus using micro-CT bone parameters, and to quantify and determine the localization of osteoclasts and RANKL during orthodontic tooth movement with corticision and 2 distinct magnitudes of applied force.

Aim 3: To identify, and quantify the severity of root resorption occurring among the experimental groups

\section{Chapter III: Materials and Methods}

\section{A. Experimental Animals}

Young (6 week old), male, Wistar rats (Charles River, body weight 150-250g) were used for the experiments. The animals were housed under standard vivarium conditions, and fed with standard powdered food (equal to standard rat chow) provided by the Animal Care Facility and water ad libitum. The food was checked and changed every day. A standard 12 hour light and dark cycle was maintained. The animals were acclimatized for at least 1 week before initiation of the experiment.

The rats were weighed every week in order to ensure that they were eating normally and were healthy. Any rat that lost more than $20 \%$ of its weight in one week, or that experienced weight loss in two consecutive weeks, was euthanized and excluded from the study.

Upon completion of the research study, the rats were euthanized by $\mathrm{CO}_{2}$, followed by cervical dislocation. All animal experimental procedures were in compliance with the guidelines for the care and use of animals in the American Journal of Physiology and the University of Connecticut Health Center.

\section{B. Experimental Design}


All experiments were performed under an institutionally approved protocol for the use of animals in research (University of Connecticut Health Center, \#2010-668). This was an experimental study with 60 rats in total. These rats were randomly placed into four groups (15 in each group). The number of rats needed in each group to provide statistical power of (1-3) of 0.8 and a type I error of 0.05 was eleven. This value was based on a power analysis performed using data from an earlier study done by Vaziri et al. All groups received an orthodontic appliance. These groups included: (1) no corticision and $\sim 10 \mathrm{~g}$ of force; (2) no corticision and $\sim 100 \mathrm{~g}$ of force; (3) corticision at the time of appliance insertion and one week afterwards with $\sim 10 \mathrm{~g}$ of force; (4) corticision at the time of appliance insertion and one a week afterwards with $\sim 100 \mathrm{~g}$ of force. In all rats the right side served as a contralateral control. The rats were subjected to the application of orthodontic force from the maxillary left first molar to the central incisors (Figure 1A).

\section{Method for orthodontic force application}

Animals were placed under general anesthesia with xylazine $(13 \mathrm{mg} / \mathrm{kg})$ and ketamine $(87$ $\mathrm{mg} / \mathrm{kg}$ ). A low force/deflection rate closed coil nickel titanium spring delivering $\sim 10 \mathrm{~g}$ of force was used for the application of light orthodontic force. A low force/deflection rate closed coil nickel titanium spring delivering $\sim 100 \mathrm{~g}$ of force was used for the application of heavy orthodontic force. The force/deflection rate $(\mathrm{F} / \Delta)$ for the spring was determined in order to calibrate the amount of force produced by activation of the spring. For the $10 \mathrm{~g}$ springs, 2-4 grams was required to activate the springs. The springs extended with a spring constant, representing their stiffness, of $14-15 \mathrm{~g}$ per $\mathrm{mm}$. For the $100 \mathrm{~g}$ springs, $85 \mathrm{~g}$ is required to activate the spring initially. The springs extend with a spring constant of 14-17 g per mm (Figure 2). 
Prior to appliance delivery a $0.008 \mathrm{~mm}$ stainless steel (SS) ligature was threaded through the contact between the first and second left maxillary molars. A light or heavy spring was then attached to the $0.008 \mathrm{~mm}$ SS ligature around the first molar and securely ligated at the mesial surface of the maxillary first molar. Self-etching primer (Transbond Plus self etching primer, 3M Unitek) was applied to the mesio-lingual surface of the maxillary first molar, and the ligature was bonded with light-cured dental adhesive resin cement (Transbond 3M Unitek) using a commercial unit (LEDemetron 1, Dentsply). Grooves $0.5 \mathrm{~mm}$ from the gingiva were prepared on the distal surfaces of the maxillary central incisors to prevent the ligatures from dislodging due to the lingual curvature and eruption pattern of the maxillary incisors. A second $0.008 \mathrm{~mm}$ SS ligature was then placed around both maxillary incisors and the spring activated and attached to this ligature. After the ligature had been tied and cut, composite resin (Transbond XT Light Cure Adhesive Paste, 3M Unitek, Monrovia, CA) was placed over the wire to prevent slipping and gingival irritation, as well as pulpal irritation due to exposed dentin. In order to minimize the distal movement of the right incisor and reinforce the anterior anchorage, the right and left incisors were joined with composite resin and acted as a unit. Finally, the mandibular incisors were reduced to prevent appliance breakage [46]. After appliance insertion the rats were allowed to recover in the presence of an incandescent light for warmth and the animals were returned to their cages once full ambulation and self-cleansing had returned. The appliance was checked twice weekly, and additional bonding material was added if necessary. One week following initial placement of the appliance, the animals were anesthetized and the springs reactivated.

Only the left side of the maxilla was treated. The contralateral side (non-treated) served as the control for histological and micro-CT purposes. The right side was also used to evaluate the physiological distal drift of the molars. 


\section{Application of Corticision}

Corticision was applied at the time of orthodontic appliance placement and one week afterwards in corticision groups. Anesthesia was induced using xylazine $(13 \mathrm{mg} / \mathrm{kg})$ and ketamine $(87 \mathrm{mg} / \mathrm{kg}$ ). A 0.036 inch SS fabricated mouthprop, placed between the maxillary and mandibular incisors, was used to hold the rat's mouth open. Corticision was performed on the mesio-palatal aspect of the left maxillary first molar. The tip of a reinforced surgical blade (No. 11, Bard-Parker, NJ, USA) capable of making a surgical incision with a minimum thickness of $400 \mu \mathrm{m}$ was employed. The blade was positioned on the mesio-palatal gingiva $0.5 \mathrm{~mm}$ from the corresponding tooth surface at an inclination of $45^{\circ}-60^{\circ}$ to the long axis of the maxillary first molar. The blade was inserted gradually into the bone marrow penetrating the overlying gingiva, cortical bone, and cancellous bone (Figure 1B).

\section{E. Measurement of Tooth Movement and Micro-CT Analysis}

Tooth movement was measured using feeler gauges on days 4, 7,11 and 14. Measurements were carried out by a single examiner and were taken by placing the feeler gauges perpendicular to the occlusal surface interproximally between the maxillary first and second molar (Figure 1C). The amount of tooth movement was calculated as the difference between time points and was compared to measurements of the untreated contralateral side (96).

Micro-CT analysis of each animal at the conclusion of the experiment was performed by the micro-CT facility at the University of Connecticut Health Center. Scanning was performed at 55 $\mathrm{kV}$ and $145 \mathrm{~mA}$, collecting 1,000 projections per rotation at 300 millisecond integration time. Three-dimensional images were constructed using standard convolution and back projection algorithms with Shepp and Logan filtering and rendered within a $16 \mathrm{~mm}$ field of view at a 
discrete density of 578,704 voxels $/ \mathrm{mm}^{3}$ (isometric $12 \mathrm{~mm}$ voxels), and a spatial resolution of $16 \mu \mathrm{m}$.

The images were used for quantitative analysis of changes occurring in the region of the maxillary $1^{\text {st }}$ molar. Tooth movement, changes in the alveolar bone and root resorption were evaluated. The amount of tooth movement was assessed on sagittal sections taken through the center of the $1^{\text {st }}$ and $2^{\text {nd }}$ maxillary molars and measured at the interproximal heights of contour between these teeth. Changes in the alveolar bone were studied by analyzing the furcation area of the maxillary $1^{\text {st }}$ molar. The region of interest (ROI) for the alveolar bone analysis was defined vertically as the most occlusal point of the furcation to the apex of the maxillary roots, transversely it was defined as the space between the buccal and lingual cortical bone, sagittally it included fifty, twelve micron sections, beginning at the mesial root and continuing distally. Parameters studied included bone volume, tissue volume, bone volume fraction, apparent density and tissue density. Bone volume represents the volume of voxels above a specific threshold considered to represent mineralized tissue and therefore representative of bone. Tissue volume is the total volume of tissue enclosed by the region of interest. Bone volume fraction is determined by the ratio of bone volume to total volume. This parameter indicates the percentage of the total volume that is made up of bone. Tissue density defines what is considered to be bone. Apparent density represents the mean value of all voxels within the region of interest including bone and background.

\section{F. Dissection and Tissue Preparation}

After decapitation, the mandibles were removed. The maxilla was then hemisected, and cleansed of soft tissue (Figure 1B). The hemisected maxilla was placed in $10 \%$ formalin for five 
days at $4{ }^{\circ} \mathrm{C}$ with constant agitation. Following fixation, samples were decalcified in $14 \%$ EDTA for 4 weeks and then processed for standard paraffin embedding. Five $\mu \mathrm{m}$ serial sagittal sections of the paraffin embedded hemimaxillae were obtained and stained with routine hematoxylin and eosin stains ( $\mathrm{H}$ and $\mathrm{E})$.

\section{G. Immunohistochemistry}

Immunohistological analyses were performed on all experimental groups. Tissue sections were deparaffinized with xylene and rehydrated with decreasing concentrations of ethanol. Following rehydration in deionized water, the sections were treated with $0.3 \%$ peroxide for 25 minutes to block endogenous peroxidase activity. In order to unmask the specific antigen, tissues were incubated in $1 \mathrm{x}$ citrate buffer $\left(\mathrm{pH} \mathrm{6.0)}\right.$ at $60^{\circ} \mathrm{C}$ overnight. The following day, tissues were washed in PBS and blocked with $10 \%$ normal goat serum in $1 \%$ bovine serum albumin (BSA) for $2 \mathrm{hrs}$. Incubation with the primary antibody was performed at $4^{\circ} \mathrm{C}$ overnight using rabbit polyclonal anti RANKL antibody at concentration 1:400 in 1\% BSA (ab9957, Abcam, Cambridge MA). The next day, tissues were washed in PBS and incubated with biotinylated goat anti rabbit secondary antibody at concentration 1:300 for 45 minutes. Elite ABC reagent (VectaStain, Vector Laboratories) was applied for $30 \mathrm{~min}$, washed thoroughly and developed with DAB (Vector Laboratories). Sections were counterstained with Harris hematoxylin for 15 seconds.

Paraffin sections were stained for tartrate-resistant acid phosphatase (TRAP) activity using an acid phosphatase leukocyte kit (Sigma Chemical, St Louis MO) according to the manufacturer's instructions. Osteoclasts were considered as TRAP positive multinucleated cells $(2+$ nuclei) and were counted on the alveolar bone surface of the compression side of the disto- 
buccal root. Histomorphometry analyses were carried out using Osteomeasure Software (Osteometrics Inc, Decatur GA). Three sections that revealed the most pulp structure (mid-root sections) were used for measurements and their means were used for statistical tests. Six animals were analyzed for each experimental group.

The area for measurement on the alveolar bone was identified as a square parallel to the sagittal axis of the distobuccal root with a width that was half of the average width of the distobuccal root and the length extending from the bifurcation to the end of the apex. Osteoclast surface was determined as the surface of an active osteoclast touching the alveolar bone and then divided by total bone surface per defined area.

Odontoclasts were considered as TRAP-positive multinucleated cells on the dentin surface and were counted on the mesial surface of the distobuccal root in the line starting from the bifurcation to the end of the apex of the distobuccal root. Odontonclast surface was considered as the surface of an active odontoclast touching the dentin and then divided by total dentin surface that extends from bifurcation to the end of the apex.

Root resorption was evaluated by identifying discontinuities along the mesial suface of the distobuccal root on histological sections. Points were placed at the margins of each break in continuity of the roots' surface, and then connected. The enclosed area was considered a resorptive crater. The areas of each crater were calculated and then summed for each section using Osteomeasure Software (Osteometrics Inc, Decatur GA).

\section{H. Statistics}

Statistical analyses were carried out using GraphPad Prism. (GraphPad Software Inc, La Jolla CA). Statistical significance of differences among means was determined by non- 
parametric, unpaired t tests or a non-parametric one way ANOVA test with a Bonferroni posttest. Significance was accorded when $\mathrm{p}<0.05$.

\section{Chapter IV: Results}

During our study, 2 animals died prematurely and were removed from the study. The first animal was lost at the beginning of the project due to an accidental overdose with anesthetic prior to having tooth movement measured on day 11. Care was taken thereafter to carefully administer the amount of ketamine. No other animals were lost as a result of anesthetic administration. The second animal \#30 was found dead in its cage. The cause of this animal's death could not be determined. Additional animals were added to the experiment to replace these animals, ensuring all groups were equal in number.

\section{A. The effect of corticision and force magnitude on tooth movement}

To assess the effect of corticision coupled with either a high or low force on the amount of tooth movement occurring over an observational period of two weeks, the distance between the maxillary first and second molars was measured on days 4, 7, 11 and 14 using feeler gauges. At the end of the experiment measurements between the first and second molars at the height of contour were also taken using micro-CT to quantify the amount of maxillary first molar mesialization.

Feeler gauge measurements used to measure the mean amount of maxillary first molar mesialization of the four experimental groups were recorded for each of the time points (days 4 , $7,11,14)$. On day four the mean amount of maxillary left first molar mesialization among the heavy force $(\sim 100 \mathrm{~g})$ groups with and without corticision was $0.53 \pm 0.07 \mathrm{~mm}$ and $0.50 \pm 0.06$ $\mathrm{mm}$, respectively. The low force $(\sim 10 \mathrm{~g})$ groups with and without corticision showed $0.44 \pm$ 
$0.08 \mathrm{~mm}$ and $0.39 \pm 0.07 \mathrm{~mm}$ of first molar mesialization, respectively. An ANOVA comparison between groups at day four showed a statistically significant difference existed between groups $(\mathrm{p}=0.000)$. A Bonferroni post hoc test (Figure 3) revealed that the amount of first molar mesialization recorded for the light force group without corticision was significantly less than the heavy force groups with and without corticision.

On day seven the mean amount of maxillary left first molar mesialization measured for the heavy force $(\sim 100 \mathrm{~g})$ groups with and without corticision was $0.75 \pm 0.05 \mathrm{~mm}$ and $0.68 \pm 0.10$ $\mathrm{mm}$, respectively. The low force $(\sim 10 \mathrm{~g})$ groups with and without corticision were measured as having $0.66 \pm 0.11 \mathrm{~mm}$ and $0.60 \pm 0.011 \mathrm{~mm}$, respectively. An ANOVA comparison between groups at day seven continued to show significance $(\mathrm{p}=0.009)$. A Bonferroni post hoc test (Figure 3) demonstrated that the difference in the amount of first molar mesialization was significantly less in the light force group without corticision versus the heavy force group with corticision.

On day eleven the mean amount of maxillary left first molar mesialization in the heavy force $(\sim 100 \mathrm{~g})$ groups with and without corticision was $0.84 \pm 0.09 \mathrm{~mm}$ and $0.80 \pm 0.13 \mathrm{~mm}$, respectively. The low force $(\sim 10 \mathrm{~g})$ groups with and without corticision experienced $0.81 \pm 0.10$ $\mathrm{mm}$ and $0.79 \pm 0.15 \mathrm{~mm}$ of left maxillary first molar mesialization, respectively. An ANOVA comparison showed no significant differences between the groups $(\mathrm{p}=0.812)$ (Figure 3$)$.

On day fourteen the mean amount of maxillary left first molar mesialization in the heavy force $(\sim 100 \mathrm{~g})$ groups with and without corticision was $0.96 \pm 0.12 \mathrm{~mm}$ and $0.86 \pm 0.21 \mathrm{~mm}$, respectively. The low force $(\sim 10 \mathrm{~g})$ groups with and without corticision had $0.93 \pm 0.08 \mathrm{~mm}$ and 
$0.84 \pm 0.21 \mathrm{~mm}$, respectively. No significance was noted between groups following an ANOVA comparison $(\mathrm{p}=0.314)($ Figure 3$)$.

At the end of the fourteen days all animals were sacrificed and the maxillae hemisected and cleansed of soft tissue. The hemisected maxillae were then submitted for micro-CT analysis. Maxillary molar mesialization was evaluated by measuring the distance between the distal height of contour of the maxillary first molar and the mesial height of contour of the maxillary second molar (Figure 1 C).

Micro-CT analysis performed on hemimaxillae of animals receiving a light force $(\sim 10 \mathrm{~g})$ without corticision for fourteen days showed that the mean amount of maxillary first molar mesialization was $0.627 \pm 0.271 \mathrm{~mm}$ on the loaded side and $0.150 \pm 0.224 \mathrm{~mm}$ on the unloaded side. The mean amount of maxillary first molar mesialization found in animals receiving light force $(\sim 10 \mathrm{~g})$ plus corticision was $0.602 \pm 0.251 \mathrm{~mm}$ on the loaded side and $0.072 \pm 0.102 \mathrm{~mm}$ on the unloaded side.

When the heavy force $(\sim 100 \mathrm{~g})$ group without corticision was studied using micro-CT the mean amount of maxillary first molar mesialization was $0.673 \pm 0.334 \mathrm{~mm}$ on the loaded side and $0.060 \pm 0.173 \mathrm{~mm}$ on the unloaded side. In the heavy force $(\sim 100 \mathrm{~g})$ group plus corticision the mean amount of maxillary first molar mesialization was $0.6631 \pm 0.2553 \mathrm{~mm}$ on the loaded side and $0.029 \pm 0.054 \mathrm{~mm}$ on the unloaded side.

To evaluate statistically significant differences in the amounts of tooth movement between the experimental groups, group to group comparisons were made using an ANOVA test. The results of these tests found that following sacrifice on day fourteen there were no significant differences in the final amounts of tooth movement observed $(\mathrm{p}=0.9354)$ (Figure 4). 
To evaluate the similarity of our measurement methods, day fourteen values using feeler gauges and micro-CT were matched for each animal and compared using paired t-tests (Figures $5,6,7,8)$. Feeler gauge measurements were significantly greater than micro-CT measurements for the light force group $(\mathrm{p}=0.0016)$ (Figure 5), light force with corticision group $(\mathrm{p}=0.0074)$ (Figure 6), heavy force group $(\mathrm{p}=0.0205)($ Figure 7$)$ and heavy force with corticision group $(\mathrm{p}=$ 0.0005) (Figure 8).

\section{B. The effect of corticision and force magnitude on the alveolus}

To evaluate the effects of a high or low force on the alveolus with or without corticision micro-CT images of the maxillary first molars were obtained for analysis. Within each experimental group, side to side comparisons were performed to evaluate difference between the loaded and unloaded sides using paired T-tests. When differences in bone volume fraction were evaluated, all of the groups with the exception of the light non-corticision group $(\mathrm{p}=0.1054)$ showed statistically significant differences between the experimental and control sides. Among the groups demonstrating statistically significant differences in bone volume fraction (light with corticision, $\mathrm{p}=0.0077$; heavy, $\mathrm{p}=0.018$; and heavy with corticision, $\mathrm{p}=0.0326$ ) all of the experimental sides showed a decrease in bone volume fraction when compared to their respective contralateral side (Figure 9).

When side by side comparisons of apparent density (light, $\mathrm{p}=0.049$; light with corticision, $\mathrm{p}$ $=0.0026$; heavy, $\mathrm{p}=0.0127$; heavy with corticision, $\mathrm{p}=0.0155$ ) and tissue density (light, $\mathrm{p}=$ 0.0145; light with corticision, $\mathrm{p}=0.0018$; heavy, $\mathrm{p}=0.0028$; heavy with corticision, $\mathrm{p}=0.003$ ) were made within each group both parameters were found to be statistically significant. Among the statistically significant findings for tissue density and apparent density the experimental sides 
all showed decreased values when compared to their respective contralateral sides (Figures 10 and 11).

Bone volume comparisons made within each group showed no significant differences. Total volume comparisons were non-significant with the exception of the light corticision group ( $p$ $=0.029)$. In the light corticision group total volume was significantly greater on the experimental side.

When intergroup comparisons of alveolar parameters were made using ANOVA there were no statistically significant findings made for BVF $(p=0.6564)$, apparent density $(p=0.9101)$ or tissue density $(\mathrm{p}=0.5941)$ (Figures 12, 13, 14).

BVF values were also calculated using histomorphometry (Figure 15). When intergroup comparisons of these BVF values were made using ANOVA there was a statistically significant difference among the groups $(\mathrm{p}=0.001)$. A Bonferroni post hoc test showed that the light force group (21.25 \pm 8.916) had significantly less BVF compared with the other groups (light with corticision $57.65 \pm 14.93$, heavy $57.09 \pm 16.74$, heavy with corticision $60.46 \pm 14.08$ ) (Figure $16)$.

\section{Correlation between bone volume fraction and the amount of orthodontic tooth movement}

To investigate whether the relationship between quantity of bone present in the region of interest at the end of the experiment was correlated with the amount of orthodontic tooth movement observed, a Spearman's nonparametric correlation coefficient $(\rho)$ was calculated for each of the groups. A Spearman correlation coefficient $(\rho)$ is a nonparametric measure of the strength and direction of association that exists between two variables measured. With $\rho$ values 
greater than zero indicating a positive relationship, those less than zero demonstrating a negative relationship and those equaling 1 or -1 representing perfect positive or negative relationships respectively.

When $\rho$ values evaluating the relationship between bone volume fraction and tooth movement were calculated for each of the groups weak to moderate linear relationships were found. A moderate positive correlation was found to exist in the heavy force group with $\rho$ equal to 0.527 . In the light force group and the light force with corticision group it was found that weak positive linear relationships exist with $\rho$ values of 0.248 and 0.358 respectively. In the heavy force group with corticision a weak negative correlation was found with a $\rho$ value of -0.012 .

\section{The effect of corticision and force magnitude on osteoclasts}

To investigate osteoclast activity, osteoclasts were counted and osteoclast surface area was measured histologically for each of the groups (Figure 17). Among all groups, the light force group $(0.3393 \pm 0.308)$ showed the highest number of osteoclasts with the light force group with corticision $(0.2238 \pm 0.1515)$ following close behind. Both of the heavy force groups with $(0.1036 \pm 0.0716)$ and without corticision $(0.1671 \pm 0.1268)$ had roughly the same amount of osteoclasts observed. When the groups were compared using ANOVA the differences observed in osteoclast counts were found to be non significant $(\mathrm{p}=0.2437)$ (Figure 18). Osteoclast surface area measurements showed slight increases in the heavy and light forces without corticsion $(6.179 \pm 4.663$ per $\mathrm{mm}$ and $7.913 \pm 5.267$ per $\mathrm{mm})$ over the heavy and light force corticision groups $(5.032 \pm 3.899$ per $\mathrm{mm}$ and $7.787 \pm 3.59$ per $\mathrm{mm})$. However, these differences were also non significant when compared with ANOVA $(\mathrm{p}=0.684)$ (Figure 19).

\section{E. The effect of corticision and force magnitude on odontoclasts}


To investigate odontoclast activity, odontoclasts were counted on the mesial surface of the distal root of the maxillary left first molar. Odontoclast numbers were highest in the light force group without corticision $(5.044 \pm 3.376)$ and the heavy force group with corticision $(4.767 \pm$ 3.099). Odontoclast numbers were least in the heavy force group without corticision (3.472 \pm 3.116) and the light force group with corticision $(2.267 \pm 3.443)$. When the groups were compared using ANOVA the differences among the groups were found to be non-significant $(\mathrm{p}=$ 0.4925) (Figure 20). The odontoclast surface area mirrored the trend found when the odontoclasts were counted. Odontoclast surface area was greatest in the heavy force group with corticision $(0.1034 \pm 0.06674$ per $\mathrm{mm})$ and the light force group without corticision $(0.09691 \pm$ 0.06517 per $\mathrm{mm}$ ). Odontoclast surface area was least in the heavy force group without corticision $(0.07205 \pm 0.06672 \mathrm{~mm})$ and the light force group with corticision $(0.0414 \pm 0.05317$ per $\mathrm{mm}$ ). Differences in odontoclast surface area was also found to be non significant when compared with ANOVA $(\mathrm{p}=0.3992)($ Figure 21).

\section{F. The effect of corticison and force magnitude on RANKL expression}

To study the effect of corticision and force magnitude on osteoclast and odontoclast differentiation and activation, the expression of receptor activator of nuclear factor kappa $\beta$ ligand (RANKL) was studied (Figure 22). RANKL expression was found to be greatest in the heavy force group without corticision $(11.02 \pm 2.37)$ and the light force group with corticision $(9.79 \pm 8.944)$. RANKL expression was lowest in the light force group without corticision (7.054 \pm 3.334$)$ and heavy force group with corticision $(4.643 \pm 2.048)$. When the groups were compared using ANOVA the differences among the groups were found to be non-significant $(\mathrm{p}=$ 0.4535) (Figure 23). 


\section{G. The effect of corticision and force magnitude on root resorption}

The effect of corticision and the two distinct force levels on the root surface was studied histomorphometrically (Figure 24). When root resorption area was calculated for the four experimental groups the mean amount of root resorption area was found to be lowest in the corticision groups (light force with corticision $0.0165 \pm 0.01713 \mathrm{~mm}^{2}$ and heavy force with corticision $0.01453 \pm 0.01077 \mathrm{~mm}^{2}$ ) and greatest in the non corticision groups (light force without corticision $0.0379 \pm 0.0173 \mathrm{~mm}^{2}$ and heavy force without corticision $0.02291 \pm 0.01689$ $\mathrm{mm}^{2}$ ). When differences between the groups' means were compared with ANOVA the differences were found to be non-significant $(p=0.0838)$ (Figure 26). Interestingly, when the groups were combined based on the presence or absence of corticision, and the means were compared with an unpaired t-test, a significant difference in means $(\mathrm{p}=0.0395)$ was found between the force group without corticision $\left(0.02955 \pm 0.01797 \mathrm{~mm}^{2}\right)$ compared to the force group with corticision $\left(0.01551 \pm 0.01368 \mathrm{~mm}^{2}\right)$ (Figure 27$)$.

\section{Chapter V: Discussion}

Historically, orthodontic treatment has been considered a gradual process requiring on average between eighteen to twenty-four months for completion. Increased length of orthodontic treatment has been cited as a point of patient dissatisfaction and has also been linked with multiple unwanted negative outcomes. Although slight, as with any medical or dental intervention, choosing to pursue orthodontic treatment is not without the risk of unwanted side effects. These risks include an elevated chance of developing caries (2), periodontal disease (3, 4), root resorption (5) and pulpal reactions (6). As is expected, as treatment duration increases so does the probability of experiencing one or more of these negative outcomes. Therefore, the 
ability to reduce treatment times would be beneficial to our patients' health and well being, as well as hold the potential to boost patient satisfaction.

Multiple methods have been attempted to enhance orthodontic tooth movement. Ultimately, velocity of tooth movement is regulated by bone turnover, bone density and the degree of hyalinization of the PDL in response to the forces being applied. Efforts to enhance the rate of orthodontic tooth movement by targeting these factors and influencing bone metabolism can be categorized as being either pharmacologic or physical. Pharmacological methods to augment the rates of tooth movement have shown great promise. Unfortunately, unwanted side effects, as well as short comings in the targeted administration of these drugs have hampered the clinical application of pharmacological agents. Due to this, increased interest has been generated regarding physical methods aimed at expediting tooth movement.

Physical efforts of enhancing tooth movement have included a wide range of techniques. These physical approaches can be classified as force based methods, surgically based methods and alternative methods. Force based methods have focused on alterations of the magnitude and duration of applied forces to move teeth. Historically, it was felt that in order to promote the maximum rate of physiologic tooth movement the optimal force should be kept as light as possible and distributed evenly along the root's surface to minimize hyalinization, undermining resorption and irreversible root resorption $(37,38,39)$. Building on the belief of the existence of an optimal force Begg put forward the concept of a differential force theory. Begg's theory held that by applying the optimal force needed to move a specific tooth it would be possible to promote that tooth's movement while eliminating unwanted reciprocal movements of anchor segments. Contradictory reports have since called the theory of differential forces into question $(40,41,42,43,44)$ and the most efficient means of moving teeth remains controversial. It now 
appears that the "optimal force" is highly variable among patients and can be best described as the lightest force capable of producing maximal tooth movement through frontal resorption with minimal patient discomfort (35). The current body of evidence seems to suggest that at lower levels, forces elicit a dose-dependent response. However, at higher levels the rate of tooth movement is not influenced by force magnitude but is dictated by the alveolus' biologic capacity to undergo remodeling.

If the speed of tooth movement is ultimately governed by the alveolus' biologic capacity to undergo remodeling, surgical methods shown to augment the process of bone remodeling may hold the key to faster tooth movement and reduced treatment times. In 2001 Wilcko and Wilcko suggested that the increased rate of orthodontic tooth movement observed following surgical manipulation of the alveolus was the result of a regional acceleratory phenomenon (58). The regional acceleratory phenomenon, first described by orthopedist Harold Frost in 1983, was based on Frost's observation that any noxious stimulus of sufficient magnitude was capable of inducing an acceleration and domination of most ongoing normal and vital tissue processes in both soft and hard tissues as part of the body's healing process. When the insult was directed at osseous tissue the result was accelerated bone turnover and reduction in regional bone density leading to a transient state of osteopenia (75). Subsequent research has confirmed that the acceleration of tooth movement associated with surgical intervention is the result of increased localized bone turnover based on a regional acceleratory phenomenon $(94,95)$ and is in proportion to the severity of the insult (60). In an effort to develop a minimally invasive, repeatable method of inducing a RAP effect with minimal patient discomfort, Kim and Park introduced corticision. With this technique a reinforced scalpel is used as a thin chisel to separate the interproximal cortices transmucosally. Based on experiments performed in a feline 
model they found it to be an efficient means of stimulating tooth movement by inducing a RAP and accelerating bone remodeling (66). Preliminary data from a study conducted at the University of Connecticut corroborate Kim and Park's findings. In this study Vaziri et al. observed that rats subjected to corticision at the beginning of the experiment and one week after the application of force demonstrated accelerated molar mesialization compared to control animals. It is hoped that the targeted application of corticision coupled with the use of sufficient forces can promote accelerated tooth movement. The purpose of this study was to assess the effect of corticision and 2 distinct force levels ( $10 \mathrm{~g}$ vs $\sim 100 \mathrm{~g})$ on the amount of orthodontic tooth movement and changes in the alveolus in a rat model after 14 days.

To evaluate what effect a high $(\sim 100 \mathrm{~g})$ and low $(\sim 10 \mathrm{~g})$ force, with and without corticision have on orthodontic tooth movement, feeler gauge measurements and micro-CT measurements were taken between the maxillary first and second molars of the rats in each group. Feeler gauge measurements were taken on days $4,7,11$ and 14 in an effort to provide a time course of the orthodontic tooth movement that took place (Figure 3). Micro-CT measurements were made on day 14 only (Figure 4). Feeler gauge measurements demonstrated significant differences among the groups only at days 4 and $7(p=0.000$ and 0.009$)$. At day 4 it was observed that there was significantly greater tooth movement in both of the heavy force groups with $(0.53 \pm 0.07 \mathrm{~mm})$ and without corticision $(0.50 \pm 0.06 \mathrm{~mm})$ when compared to the light force only group $(0.39 \pm 0.07 \mathrm{~mm})(\mathrm{p}=0.000)$. At day 7 , significantly greater tooth movement remained only for the heavy force group with corticision $(0.68 \pm 0.10 \mathrm{~mm})$ when compared to the light force only group $(0.60 \pm 0.011 \mathrm{~mm})(\mathrm{p}=0.009)$. Feeler gauge measurements on days 11 and 14 were unremarkable. Micro-CT tooth movement measurements 
on day 14 were also non-significant (Figure 5). These findings support our first null hypothesis that there will be no difference in the amount of tooth movement among the groups.

When the values of the tooth movement measurements using micro-CT and the feeler gauges were compared, it was found that measurements were significantly different from one another, with the feeler gauge measurements over estimating the amount of tooth movement (Figure $6,7,8,9$ ). This could be explained by a number of factors. The feeler gauge measurements were plagued by multiple short comings including the inherent difficulty of taking accurate intraoral measurements on live animals, the mobility of teeth being studied due to widening of the PDL as a result of orthodontic tooth movement and the inability to take precise measurements with a limited selection of gauges. In addition the standard deviation of values around the mean using micro-CT was greater. This suggests a greater ability to accurately detect the amount of tooth movement that occurred for each animal using micro-CT. If the values obtained from the feeler gauges are used qualitatively to represent a trend, the differences present on days 4 and 7 (Figure 2) may support the findings of other studies showing the effect of corticision and corticotomies to be short lived. For instance, Baloul et al. (95) demonstrated that over a six week time course following selective alveolar decortication in rats, tooth movement was significantly enhanced only during the first week when compared to a tooth movement only group. Furthermore, Kim and Park observed that following application of corticision the majority of tooth movement occurs during the first 2 weeks (66).

Alveolar changes were studied using both micro-CT analysis and histomorphometry. Micro-CT analysis was used to study the effects of a high or low force on the alveolus with or without corticision at the furcation of the maxillary first molars. Parameters studied included bone volume, tissue volume, bone volume fraction, apparent density and tissue density. Within 
each experimental group side to side comparisons were performed to evaluate differences between the loaded and unloaded sides using paired T-tests. Significant differences existed between the experimental and control sides for all groups with respect to apparent density and tissue density, with the experimental sides all showing a decrease (Figures 10 and 11). Bone volume fraction also demonstrated a significant difference between the experimental and control sides for all of the groups, with the exception of the light non-corticision group (Figure 9). Again, the experimental sides showed decreased values compared to their contralateral controls. These intra-group findings reflect the bony remodeling occurring at the alveolus during orthodontic tooth movement $(7,24)$. When intergroup comparisons were made, no significant differences were found with respect to any of the parameters studied indicating no differences in the amount of alveolar change occurring at the furcation among the groups (Figures 12, 13, 14). This supports the lack of a significant difference in the amount of tooth movement observed among any of the groups.

Histologic sections were also used to evaluate changes occurring at the furcation area of the left maxillary first molars (Figure 15 and 17). Bone volume fraction was found to be significantly less in the light force group compared to the others $(\mathrm{p}=0.001)$ (Figure 16). This finding was different from what was observed using micro-CT. The difference between the two methods likely reflects that the region of interest using micro-CT was larger. The larger region of interest may have washed out the changes observed histologically along the distobuccal root. Osteoclast number per BVF and osteoclast surface showed no significant differences among the groups (Figures 18 and 19). Although not significant, on average osteoclast numbers and surface area were greatest for the light force group and greater when non corticision groups were compared to corticision groups. Despite not being significant, the values of the osteoclast 
parameters studied combined with the significant BVF in the light force group may indicate that whatever changes occurring in the corticision groups may have occurred early on in the experiment consistent with a short lived regional acceleratory phenomenon reported by other investigators $(64,65,94,95)$. When odontoclast number and surface were compared, no significant differences were observed among the groups (Figures 20 and 21). Differences in RANKL expression were also found to be non significant (Figure 23).

In an effort to study the effect different force levels with and without corticision had on the root surface, the size of root surface craters along the distobuccal root were calculated using our histologic specimens (Figure 24), and micro-CT surface renderings of the distobuccal root were evaluated qualitatively (Figure 25). When the groups were compared no significant differences were evident (Figure 26). However, when groups were combined based on the presence or absence of corticision there was a significant difference in the amount of root resorption present $(\mathrm{p}=0.0395)$ (Figure 27$)$, with corticision having a protective effect. It has been shown in other studies that surgical insults to the alveolus trigger a regional acceleratory phenomenon resulting in increased alveolar remodeling, thus providing an enhanced adaptive capacity of the alveolus to applied forces $(58,94)$. The protective effect corticision had in this study against root resorption provides additional evidence corticision is capable of stimulating a regional acceleratory phenomenon.

This study was not without limitations. As already mentioned, the feeler gauge measurements used to provide a time course of tooth movement were found to be inaccurate when compared with micro-CT measurements. This calls into question the significant findings found during the first week that showed increased tooth movement in the heavy force groups with and without corticision when compared to the light force only group. 
Furthermore, the application of corticision could be improved. In this study, corticision was applied with a reinforced scalpel blade on days zero and seven on the mesio-palatal aspect of the maxillary left first molar. Despite efforts to consistently apply corticision on the mesiopalatal gingiva, $0.5 \mathrm{~mm}$ from the corresponding tooth surface at an inclination of $45^{\circ}-60^{\circ}$ to the long axis of the maxillary first molar with the blade inserted gradually into the bone marrow penetrating the overlying gingiva, cortical bone, and cancellous bone, it is unknown if this was actually achieved consistently for all animals and at each time point. To allow for more consistent application it would be advisable to use some type of surgical guide to standardize the application, and a blade that would limit or indicate the depth of the incision being made. In addition, the lack of significance found between groups at the end of our experiment with regards to tooth movement and alveolar changes could have been due to the limited site of corticision application. Unlike Kim and Park, who performed corticision on the mesiobuccal, distobuccal and distopalatal aspects of feline maxillary canines (65) and mesiobuccal, distobuccal and distopalatal aspect of beagle maxillary second premolars (66) and found significant differences in both tooth movement and alveolar remodeling, our study applied corticision only on the mesiopalatal aspect of the maxillary left rat first molars.

The reactivation of springs could also have been a limitation in this study. In order to ensure all springs were active throughout the experiment they were reactivated on day seven. Based on preliminary studies of the spring characteristics prior to the experiment it was known that the springs would provide the desired amounts of force within a specific window of activation. For instance, the light and heavy springs provided $\sim 10 \mathrm{~g}$ and $\sim 100 \mathrm{~g}$ of force respectively, when activated one $\mathrm{mm}$. However, when the light and heavy springs were extended beyond this range an additional 14-15 grams of force was applied per mm. Although 
they still delivered a light and heavy force, the amount of activation would influence the behavior of the springs within each group.

Lastly, our analysis of alveolar changes and root resorption had inherent short comings. Due to the method of molar mesialization chosen, with springs attached at the level of the crowns and not at the center of resistance of these teeth we found the teeth had a tendency to tip mesially and rotate palatally. Therefore histologic analysis assuming the mesial surface of the distobuccal root represented an area of compression was only partially correct. Instead it is more likely the mesial surface of the distobuccal root included areas of both compression and tension due to the tipping and rotation that occurred. In addition our method of analyzing root resorption histologically was able to capture only a very small cross section of what was actually occurring on the surface of the root, and was unable to quantify changes occurring at the apex. Undoubtedly data obtained from micro-CT would be better suited for quantification of root resorption.

\section{Chapter VI: Conclusion}

With regards to tooth movement our feeler gauge measurement showed there were significant differences in the amount of tooth movement between groups at days four and seven. On day four, both heavy force groups showed significantly greater molar mesialization compared to the light force group without corticision. On day seven, only the heavy force group with corticision displayed significantly greater amounts of tooth movement compared to the light force only group. On days eleven and fourteen, no difference were detected among the groups using the feeler gauges. Micro-CT measurements taken at the conclusion of the experiment also showed no differences in the amounts of tooth movement. When the micro-CT and feeler gauge 
measurements at day fourteen were compared they were found to be significantly different with the feeler gauge measurement overestimating the amount of tooth movement. Based on these findings the null hypothesis that there will be no difference in the amount of tooth movement among the experimental groups with or without corticision or a light or heavy protractive force must be accepted.

The effects heavy and light forces with and without corticision had on the alveolus were studied histologically and using micro-CT. It was observed using micro-CT that light and heavy forces with and without corticision resulted in reduced tissue density and BVF compared to the contralateral control molar with the exception of BVF for the light force at the end of 14 days. Micro-CT analysis of the ROI in the alveolus showed no significant differences among the experimental groups with regards to bone volume fraction and tissue density at the end of 14 days. Histomorphometric analysis demonstrated a significant decrease in BVF in the light force group. Although not significant, on average there were more osteoclasts and a greater amount of osteoclast surface area found in the light force group at the end of 14 days. Histomorphometric analysis showed no significant differences in the number of odontoclasts or the amount of odontoclast surface area among the experimental groups at the end of 14 days. Immunohistochemistry targeting RANKL found no significant differences in the amount of RANKL present among the groups. Analysis histologically of root resorption craters among the experimental groups showed no significant differences. When the groups were combined based on the presence or absence of corticision, those receiving corticision showed significantly less root resorption compared to the force only groups. Based on our results we cannot reject our null hypothesis that there will be no difference in osteoclast activity among the groups. 
In summary, on day 14 there were no significant differences in tooth movement, microCT bone parameters or number of osteo/odontoclasts among experimental groups. Only light force caused a significant decrease in BVF observed histologically. Corticision had a protective effect against root resorption. 


\section{Chapter VII: Figures}

A

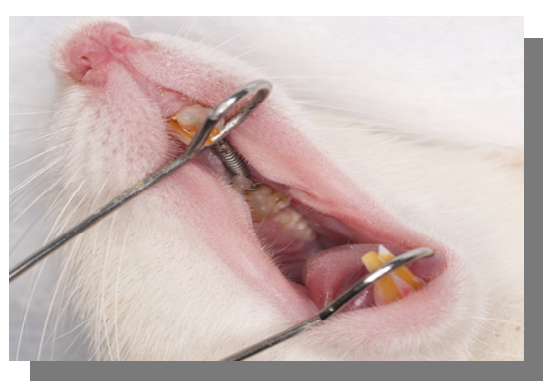

B

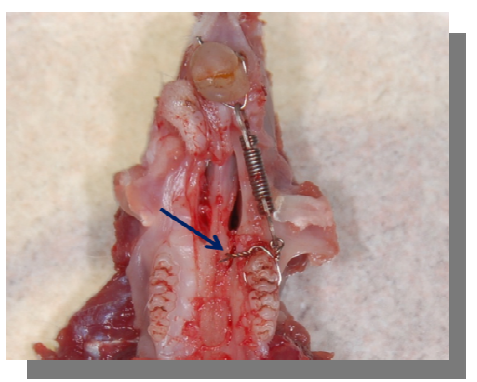

C

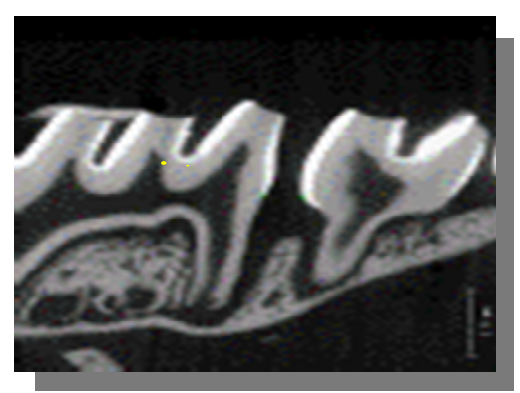

Figure 1: (A) Experimental design demonstrating orthodontic tooth movement. (B) Location of corticision application (blue arrow). (C) Micro-CT image showing displacement between the first and second maxillary second molars and ROI at the furcation of the maxillary first molar.
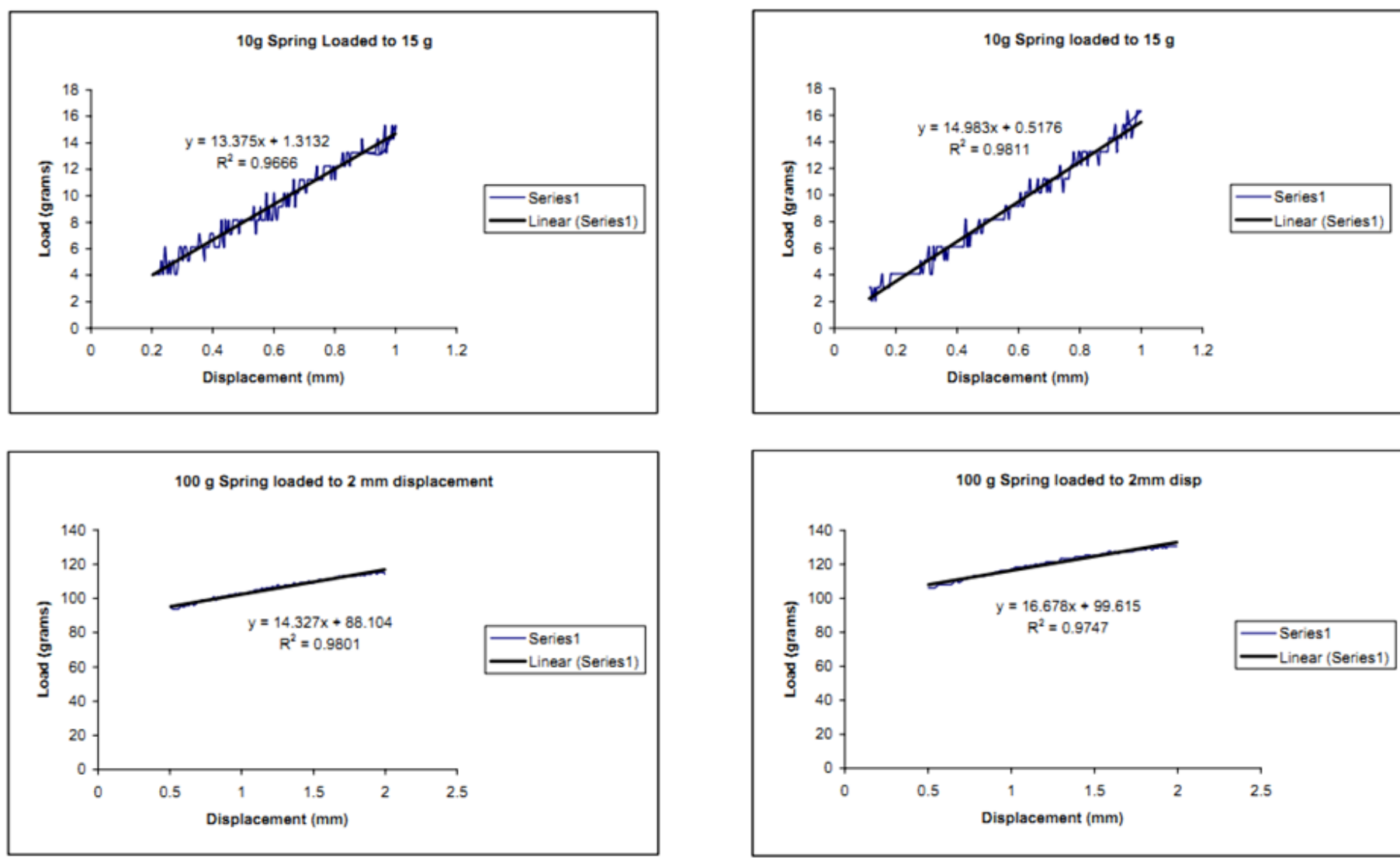

Figure 2: Spring stress strain curves for apporoximately 10 and 100 gram nickel titanium coil springs 

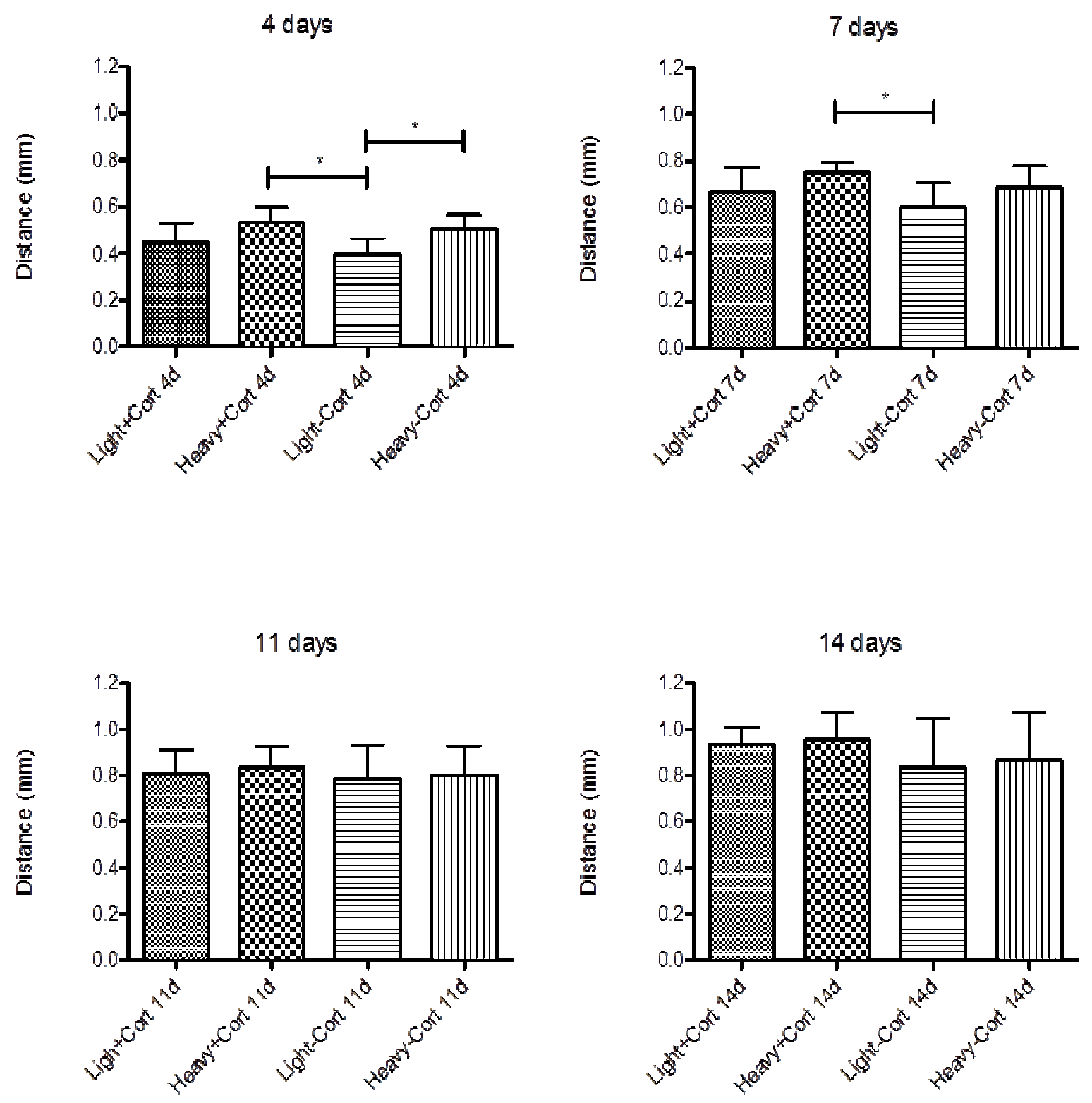

Figure 3. Comparison of $1^{\text {st }}$ molar mesialization at 4, 7, 11 and 14 days. ANOVA comparison of light $(\sim 10 \mathrm{~g})$ and heavy $(\sim 100 \mathrm{~g})$ force groups with and without corticision. Significant difference between groups is noted by $*(\mathrm{P}<0.05)$ 


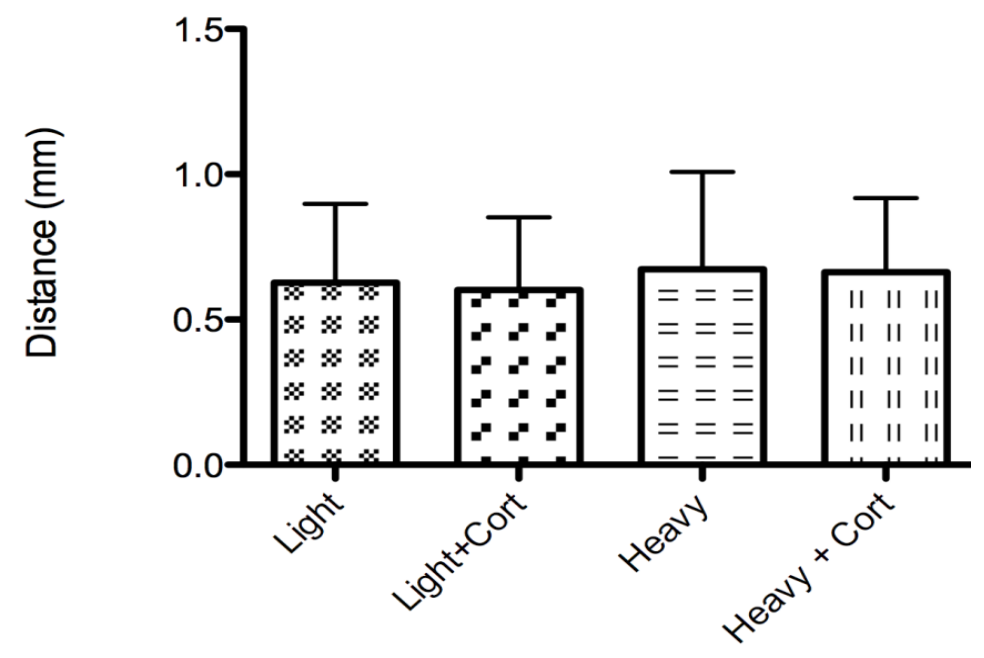

Figure 4. Comparison of $1^{\text {st }}$ molar mesialization on day 14 using $\mu \mathrm{CT}$. ANOVA comparisons of light $(\sim 10 \mathrm{~g})$ and heavy $(\sim 100 \mathrm{~g})$ force groups with and without corticision. No significant difference between groups is noted $(\mathrm{n}=11)$.

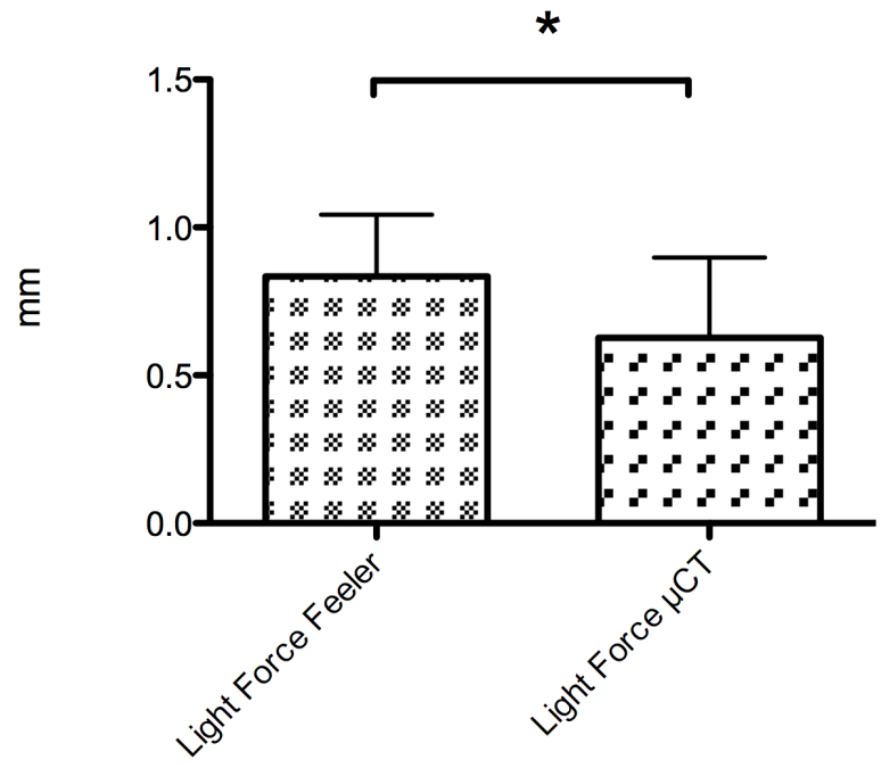

Figure 5. Comparison of feeler gauge and $\mu \mathrm{CT}$ measurements taken on the light $(\sim 10 \mathrm{~g})$ force group without corticision on day 14 using a paired t-test. Significant difference between groups is noted by * $(\mathrm{P}<0.05),(\mathrm{n}=10)$. 


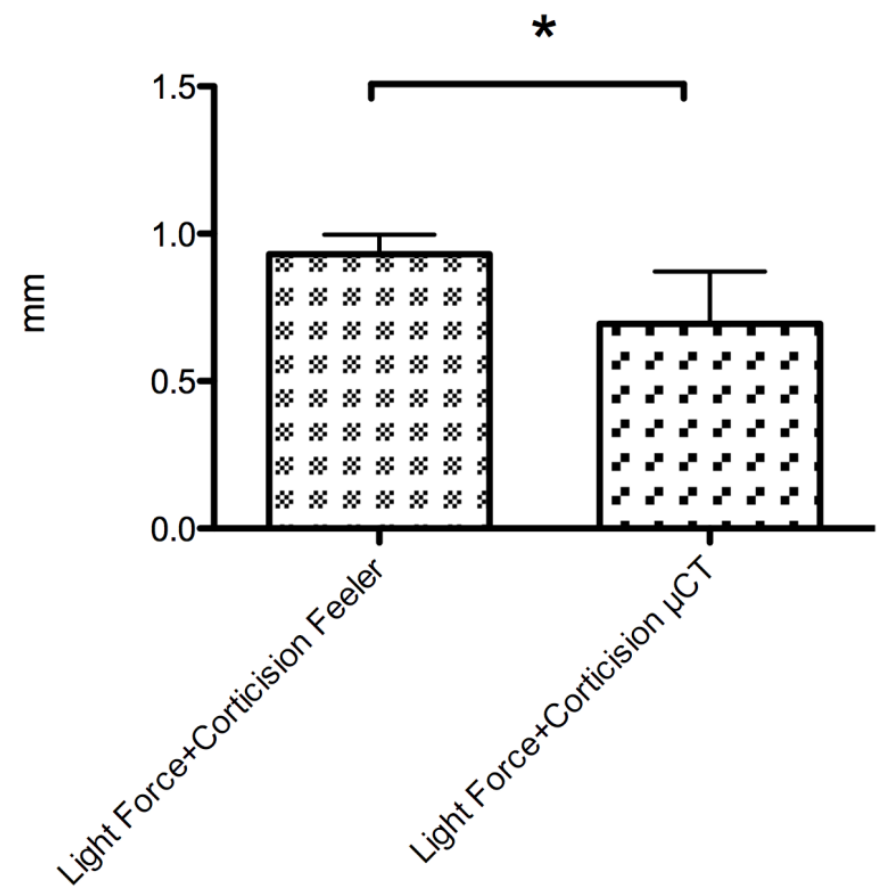

Figure 6. Comparison of feeler gauge and $\mu \mathrm{CT}$ measurements taken on the light $(\sim 10 \mathrm{~g})$ force group with corticision on day 14 using a paired t-test. Significant difference between groups is noted by $*(\mathrm{P}<0.05)$, $(\mathrm{n}=8)$.

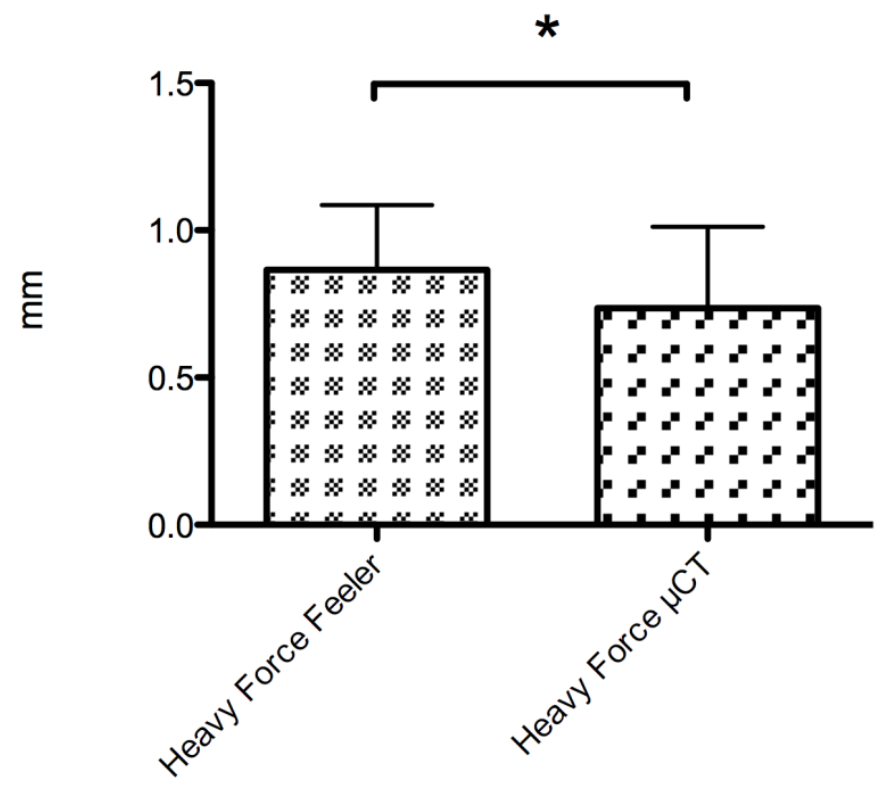

Figure 7. Comparison of feeler gauge and $\mu \mathrm{CT}$ measurements taken on the heavy $(\sim 100 \mathrm{~g})$ force group without corticision on day 14 using a paired t-test. Significant difference between groups is noted by * $(\mathrm{P}<0.05),(\mathrm{n}=10)$. 


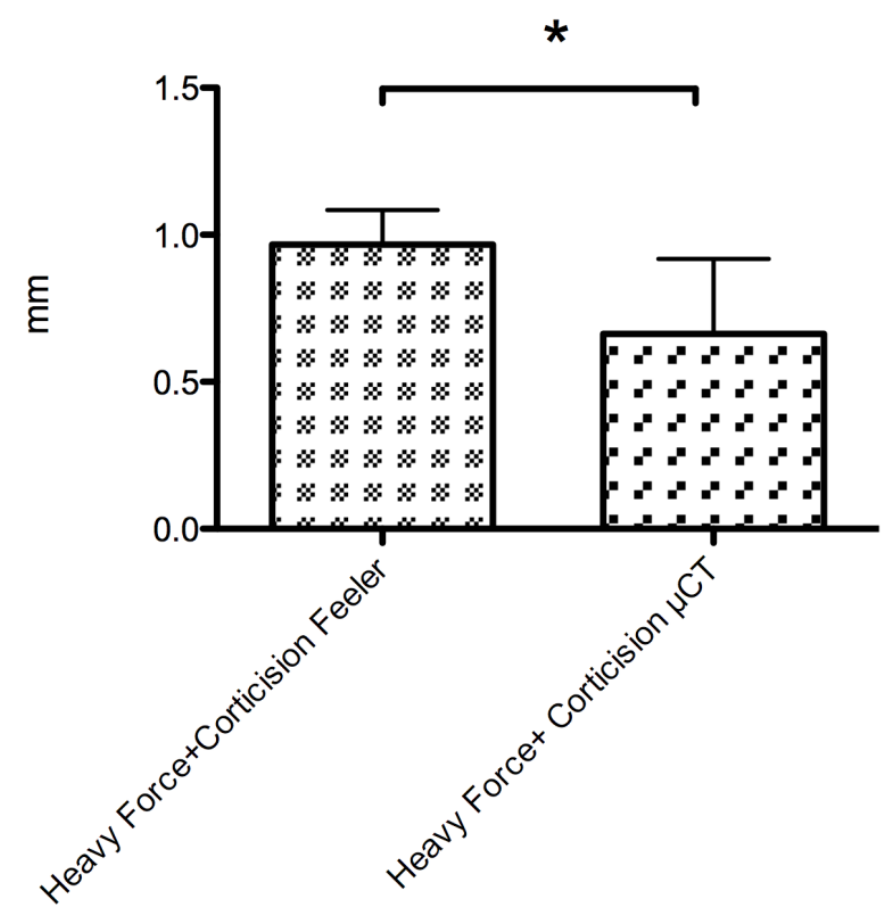

Figure 8. Comparison of feeler gauge and $\mu \mathrm{CT}$ measurements taken on the heavy $(\sim 100 \mathrm{~g})$ force group with corticision on day 14 using a paired t-test. Significant difference between groups is noted by * $(\mathrm{P}<0.05),(\mathrm{n}=10)$.

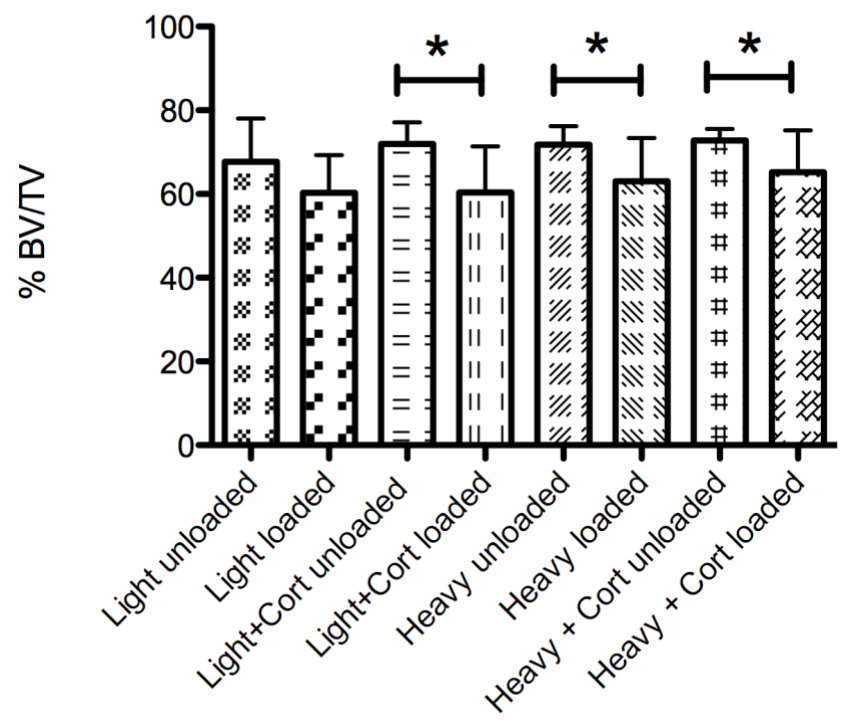

Figure 9. Comparison of intragroup bone volume fraction with the contralateral control molar on day 14 using $\mu \mathrm{CT}$. ANOVA comparisons of light $(\sim 10 \mathrm{~g})$ and heavy $(\sim 100 \mathrm{~g})$ force groups with and without corticision. Significant difference between groups is noted by $*(\mathrm{P}<0.05),(\mathrm{n}=11)$. 


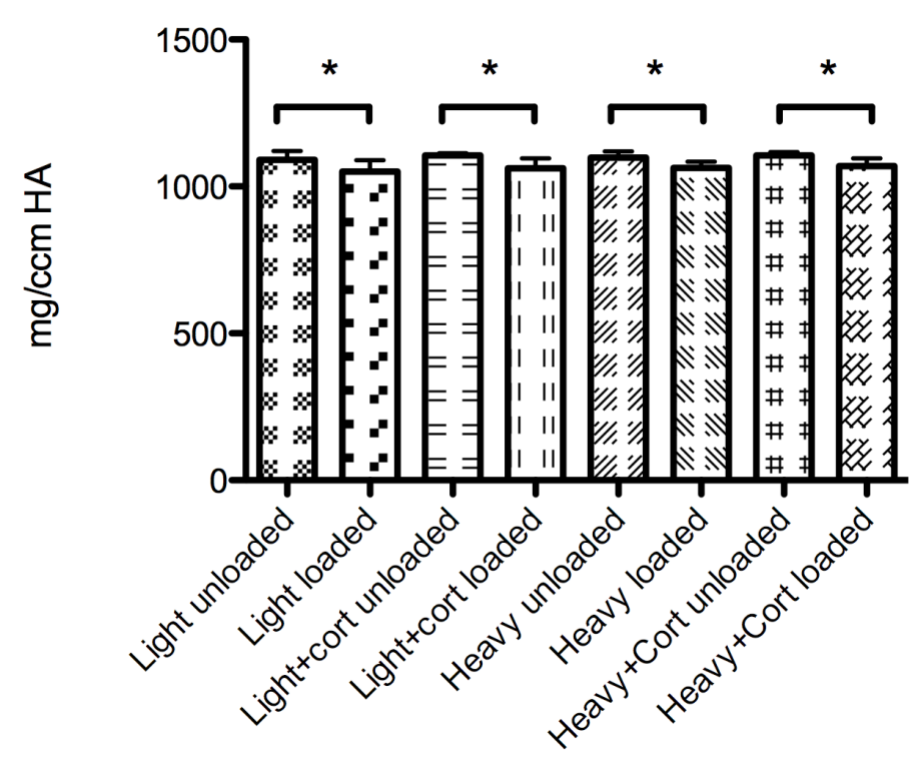

Figure 10. Comparison of intragroup tissue density with the contralateral control molar on day 14 using $\mu \mathrm{CT}$. ANOVA comparisons of light $(\sim 10 \mathrm{~g})$ and heavy $(\sim 100 \mathrm{~g})$ force groups with and without corticision. Significant difference between groups is noted by $*(\mathrm{P}<0.05),(\mathrm{n}=11)$.

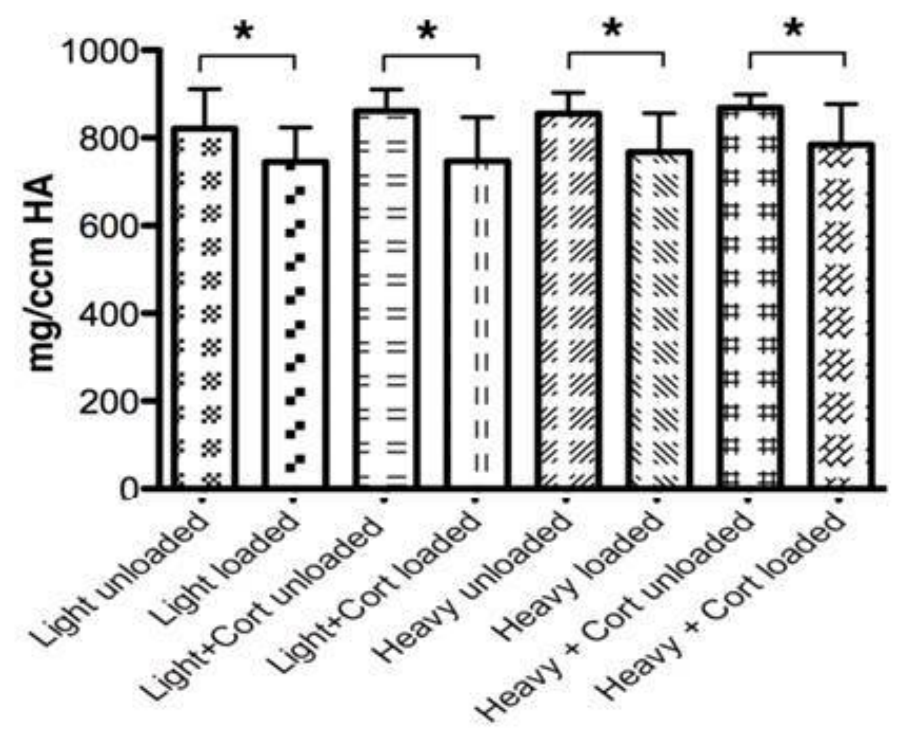

Figure 11. Comparison of intragroup apparent density with the contralateral control molar on day 14 using $\mu \mathrm{CT}$. ANOVA comparisons of light $(\sim 10 \mathrm{~g})$ and heavy $(\sim 100 \mathrm{~g})$ force groups with and without corticision. Signficant difference between groups is noted by $*(\mathrm{P}<0.05),(\mathrm{n}=11)$. 


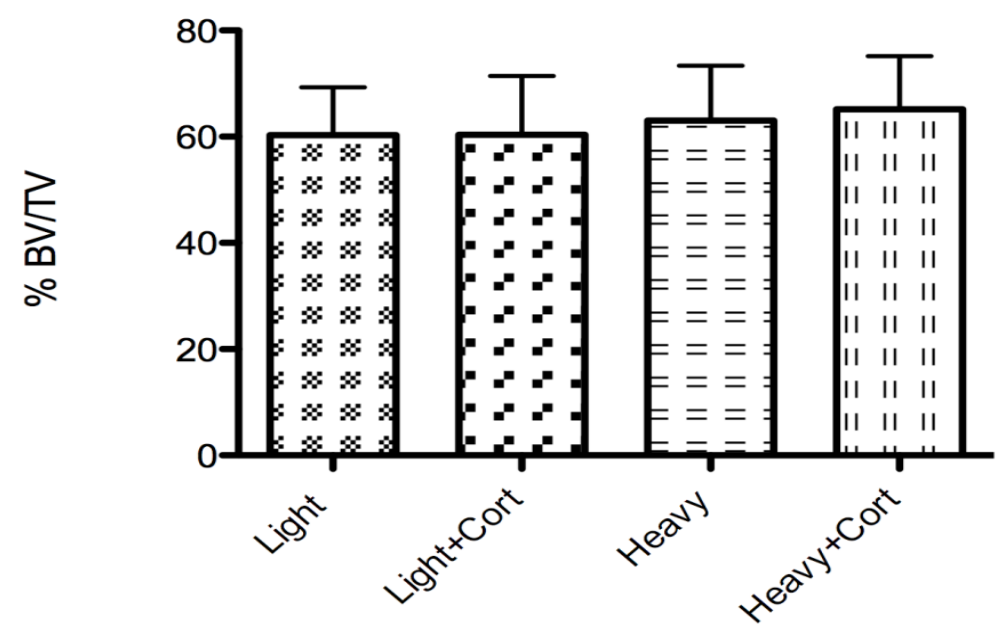

Figure 12. Comparison of intergroup bone volume fraction on day 14 using $\mu C T$. ANOVA comparisons of light $(\sim 10 \mathrm{~g})$ and heavy $(\sim 100 \mathrm{~g})$ force groups with and without corticision. No significant difference between groups noted $(n=11)$.

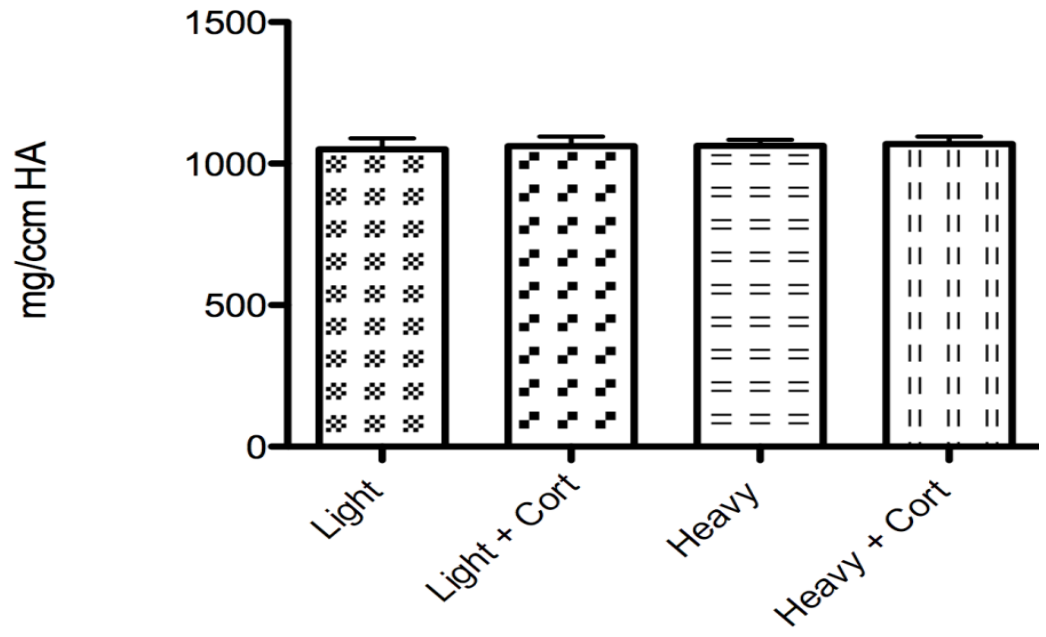

Figure 13. Comparison of intergroup tissue density on day 14 using $\mu \mathrm{CT}$. ANOVA comparisons of light $(\sim 10 \mathrm{~g})$ and heavy $(\sim 100 \mathrm{~g})$ force groups with and without corticision. No significant difference between groups noted $(\mathrm{n}=11)$. 


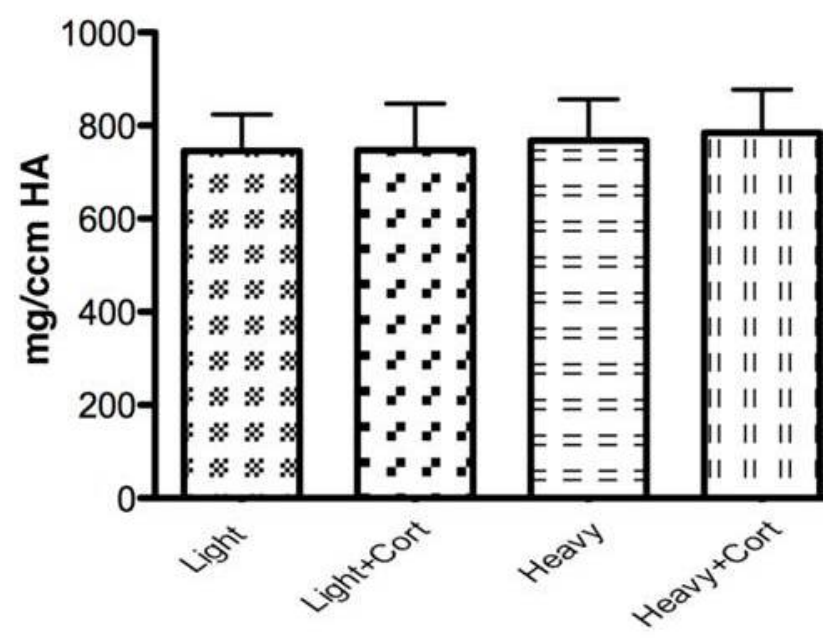

Figure 14. Comparison of intergroup apparent density on day 14 using $\mu \mathrm{CT}$. ANOVA comparisons of light $(\sim 10 \mathrm{~g})$ and heavy $(\sim 100 \mathrm{~g})$ force groups with and without corticision. No significant difference between groups noted $(\mathrm{n}=11)$.

Light Force

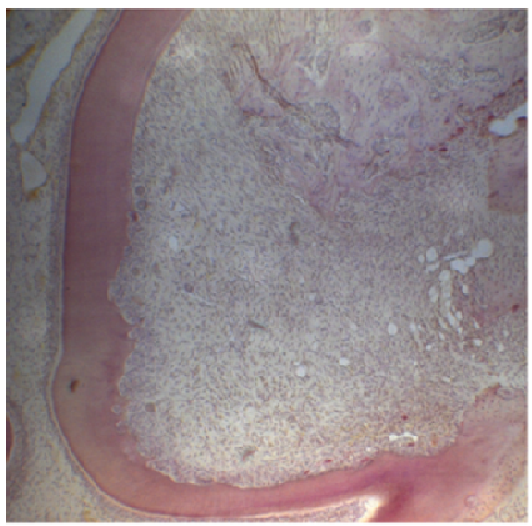

Heavy Force

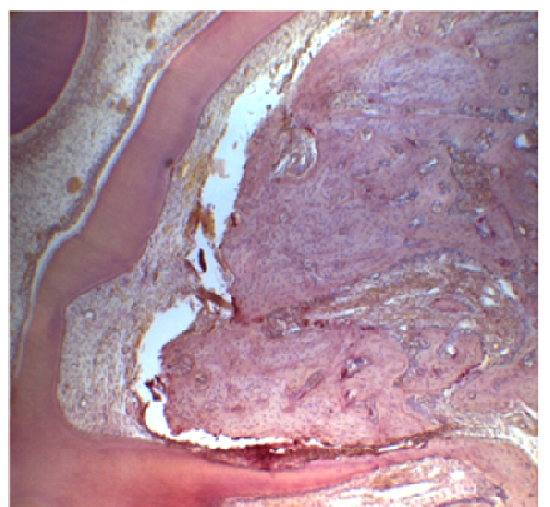

Light Force+Corticision

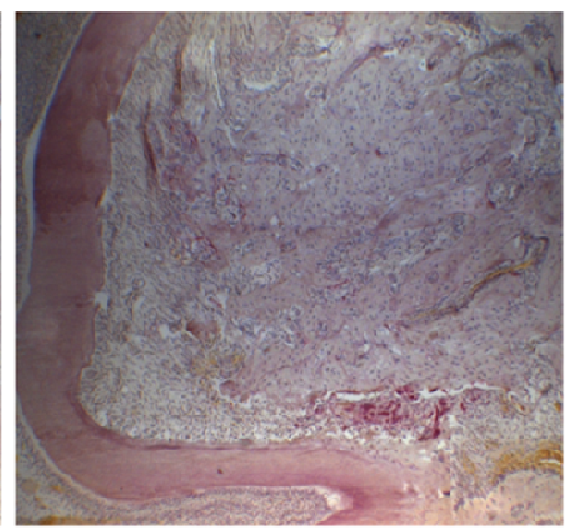

Heavy Force+Corticision

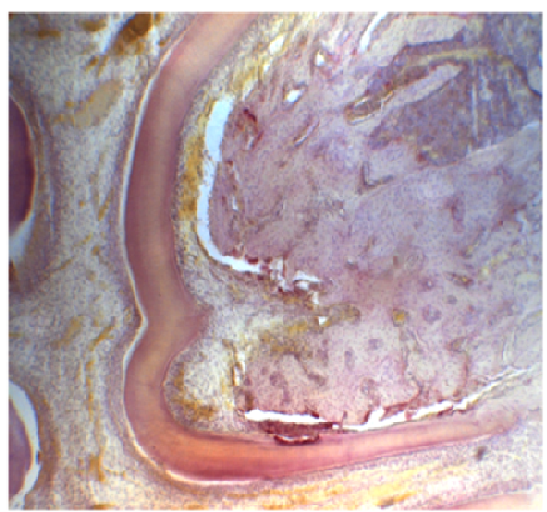

Figure 15. Histologic images capturing the ROI (furcation area and mesial aspect of the distobuccal root) used for histomorphometric analysis. 


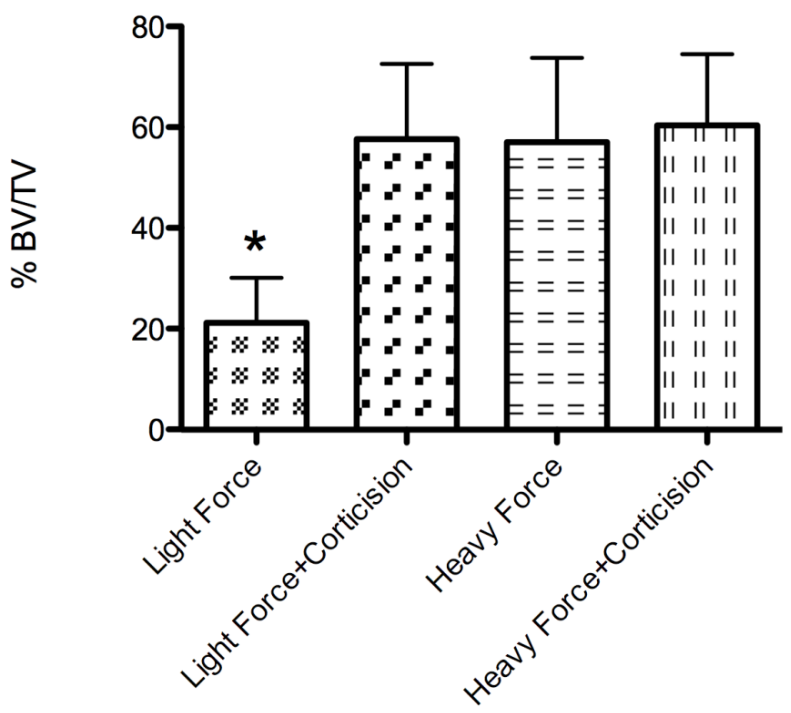

Figure 16. Comparison of intergroup bone volume fraction on day 14 using histomorphometry. ANOVA comparisons of light $(\sim 10 \mathrm{~g})$ and heavy $(\sim 100 \mathrm{~g})$ force groups with and without corticision. Significant difference between groups is noted by $*(\mathrm{P}<0.05),(\mathrm{n}=6)$.

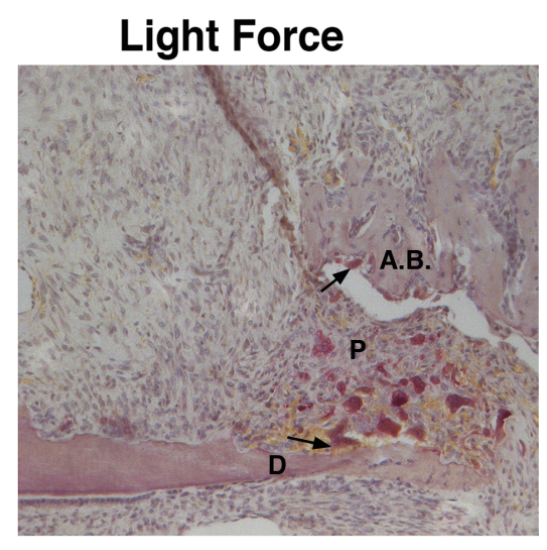

\section{Light Force + Corticision}
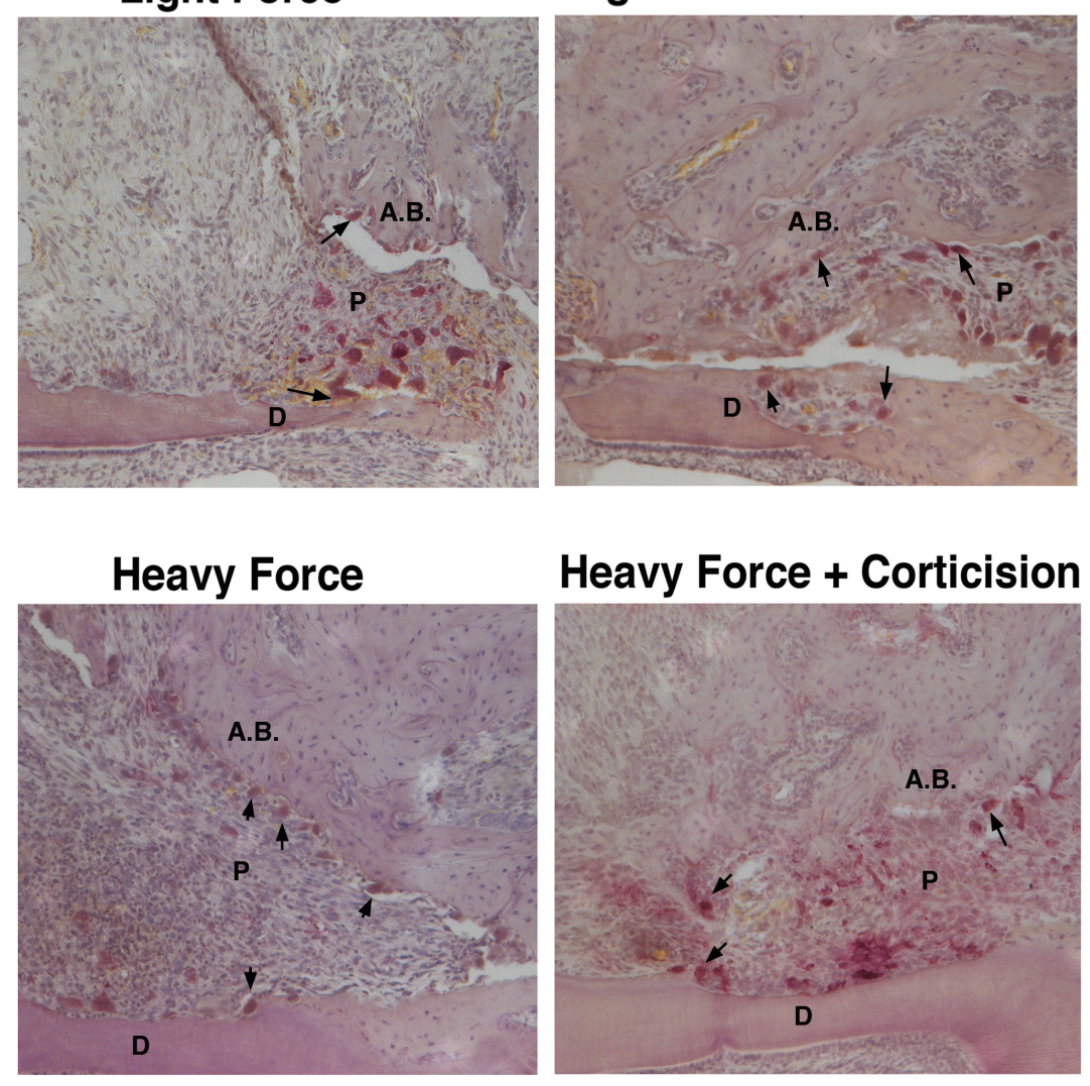

Figure 17. TRAP stained sections. Arrows point to TRAP positive cells lying on alveolar bone and dentin. 


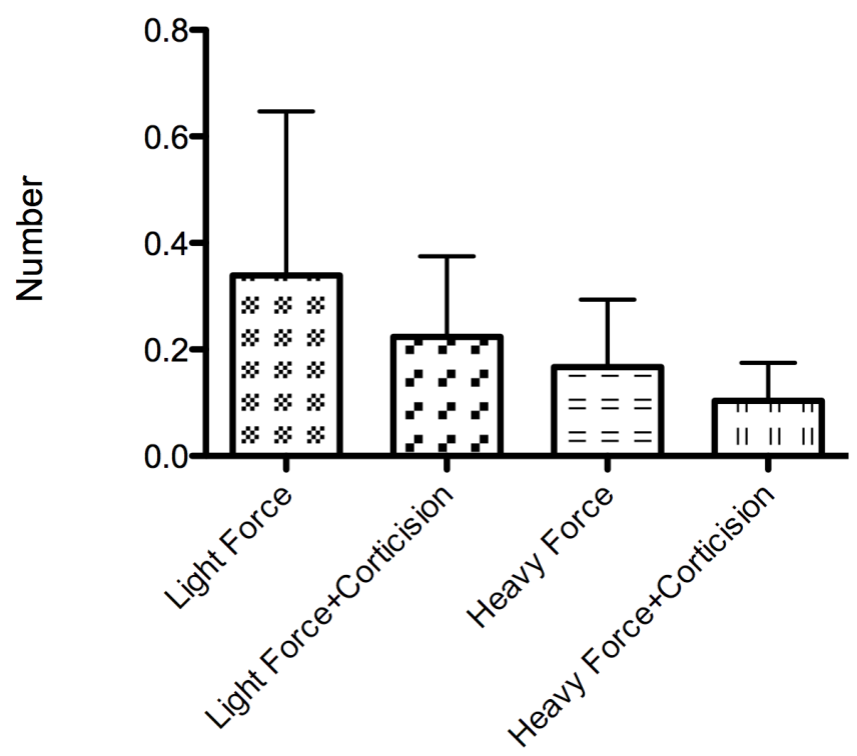

Figure 18. Comparison of osteoclast number / BVF on day 14 measured using histomorphometry. ANOVA comparisons of light $(\sim 10 \mathrm{~g})$ and heavy $(\sim 100 \mathrm{~g})$ force groups with and without corticision. No significant difference between groups noted $(n=6)$.

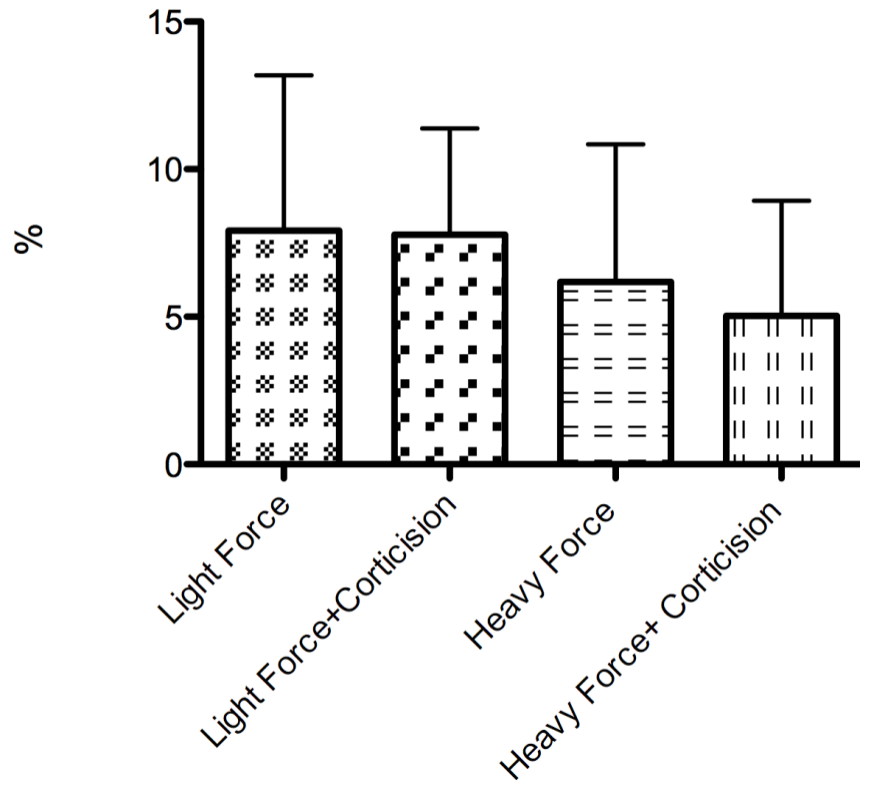

Figure 19. Comparison of osteoclast surface on day 14 measured using histomorphometry. ANOVA comparisons of light $(\sim 10 \mathrm{~g})$ and heavy $(\sim 100 \mathrm{~g})$ force groups with and without corticision. No significant difference between groups noted $(n=6)$. 


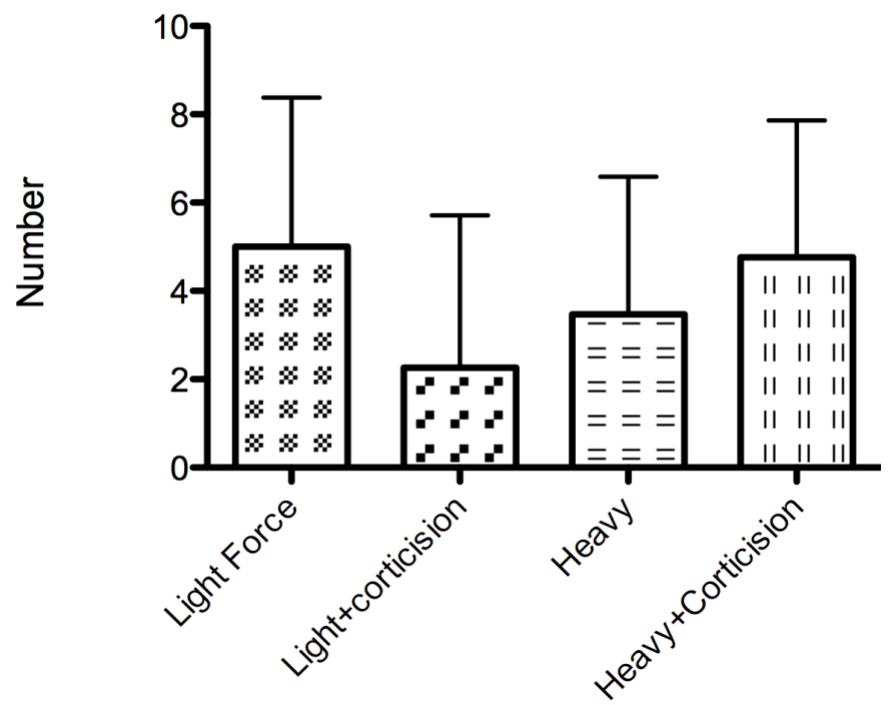

Figure 20. Comparison of odontoclast number on day 14. ANOVA comparisons of light $(\sim 10 \mathrm{~g})$ and heavy $(\sim 100 \mathrm{~g})$ force groups with and without corticision. No significant difference between groups noted $(\mathrm{n}=6)$.

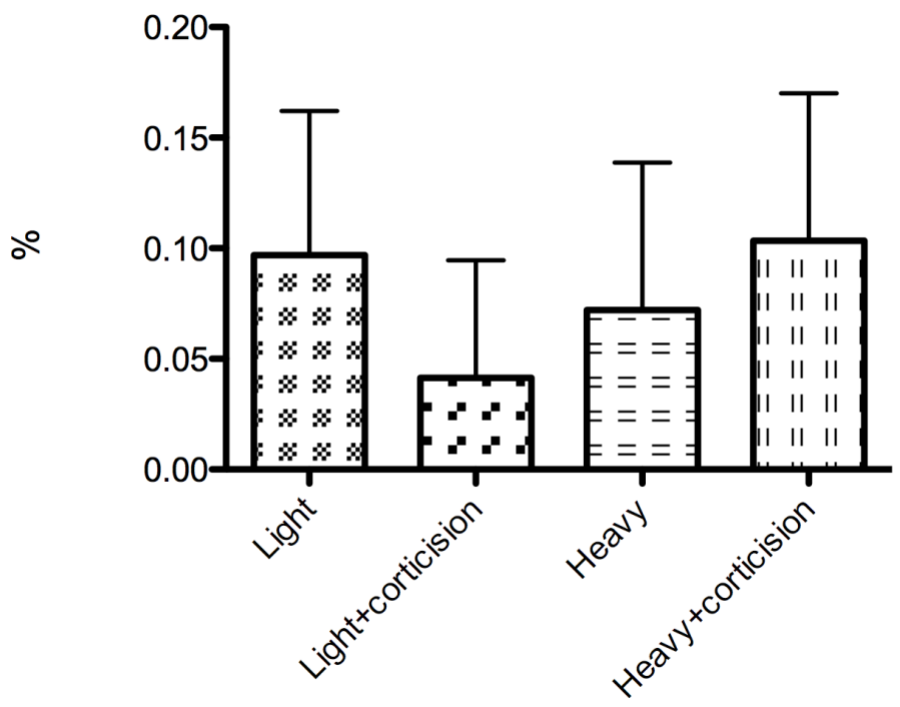

Figure 21. Comparison of odontoclast surface on day 14. ANOVA comparisons of light $(\sim 10 \mathrm{~g})$ and heavy $(\sim 100 \mathrm{~g})$ force groups with and without corticision. No significant difference between groups noted $(\mathrm{n}=6)$. 
Light Force

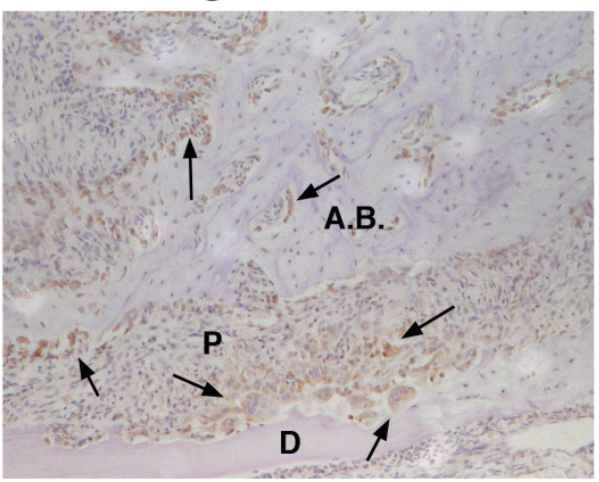

Heavy Force

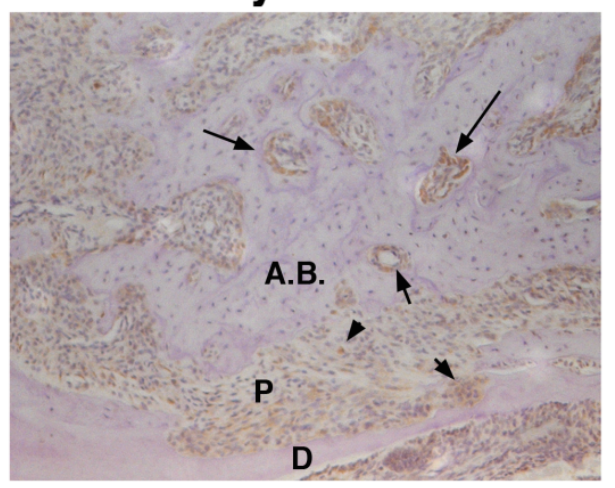

\section{Light Force + Corticision}
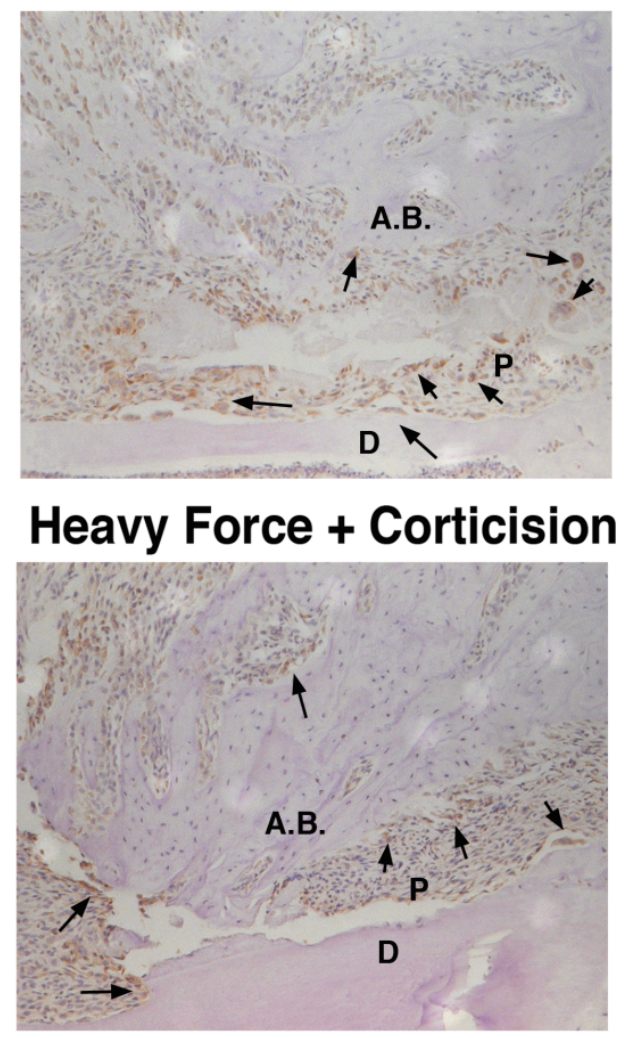

Figure 22. RANKL stained sections. Arrows point to RANKL positive cells along the alveolar bone and dentin

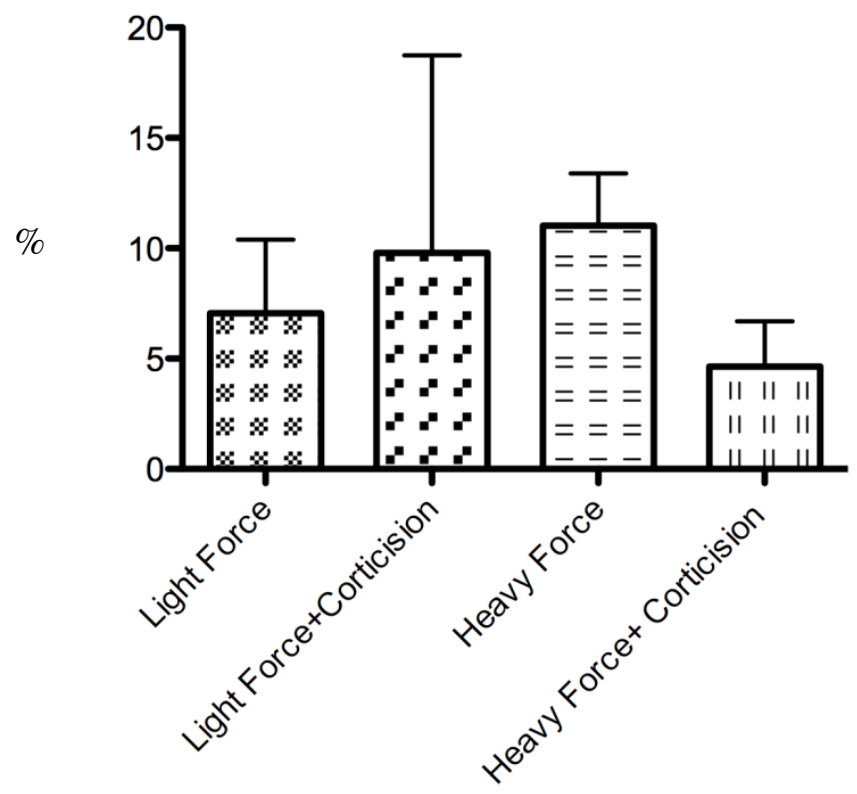

Figure 23 . Comparison of RANKL positive cells. ANOVA comparisons of light $(\sim 10 \mathrm{~g})$ and heavy $(\sim 100 \mathrm{~g})$ force groups with and without corticision. No significant difference between groups noted $(\mathrm{n}=3)$. 


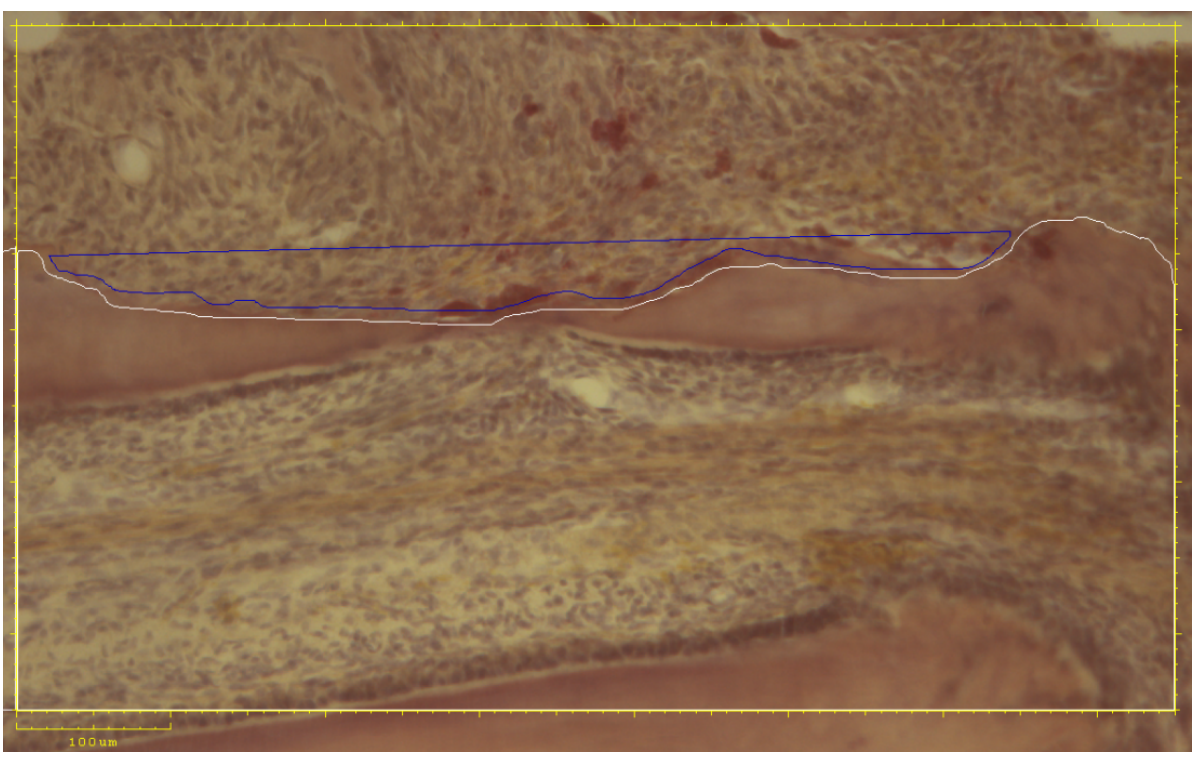

Figure 24. Evaluation of root resorption on the distobuccal root using histomorphometry.
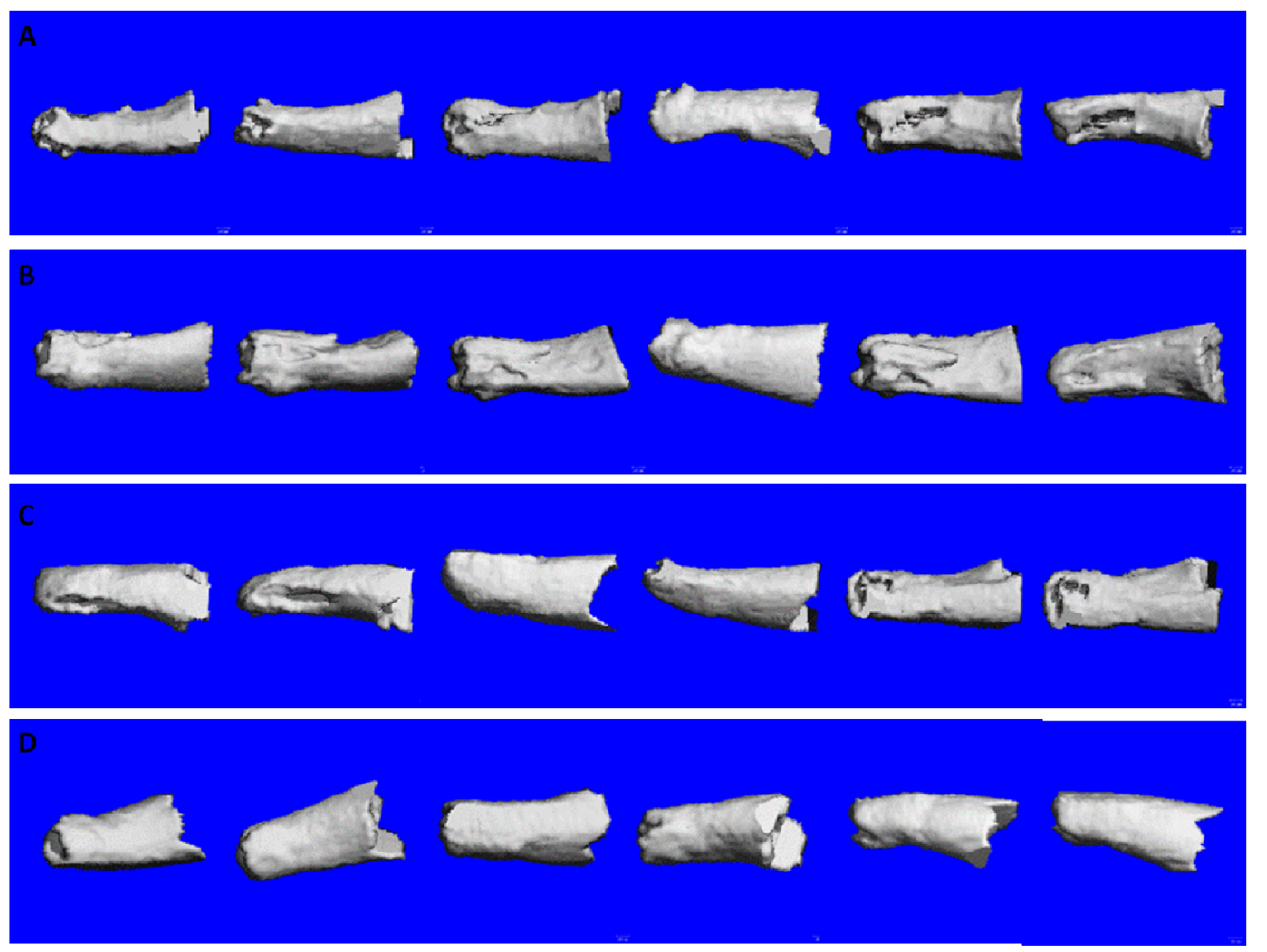

Figure 25. Micro-CT surface rendering of the distobuccal root of (A) light force without corticision (B) light surface with corticision (C) heavy force without corticision (D) heavy force with corticision. 


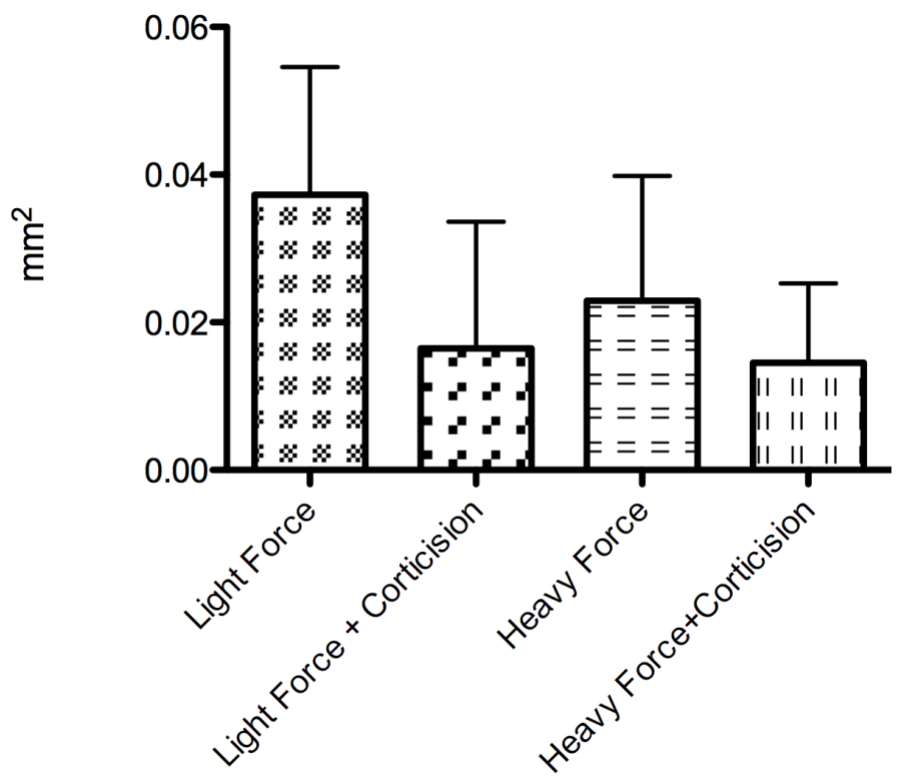

Figure 25. Intergroup comparison of root resorption area on day 14. ANOVA comparisons of light $(\sim 10 \mathrm{~g})$ and heavy $(\sim 100 \mathrm{~g})$ force groups with and without corticision. No significant difference between groups noted $(\mathrm{n}=4-5)$.

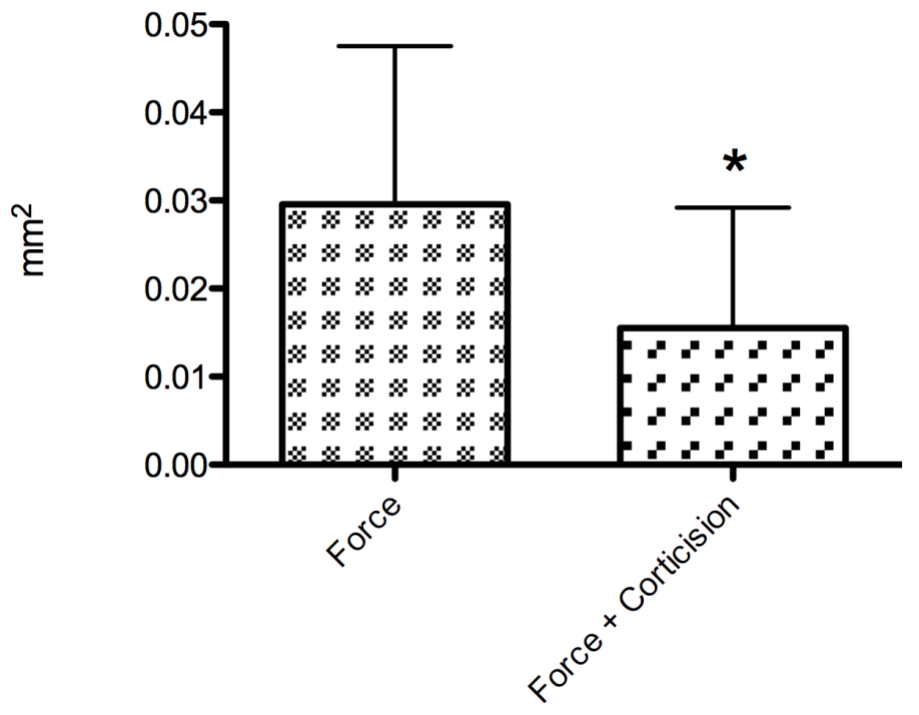

Figure 26. Intergroup comparison of root resorption area on day 14. Unpaired t-tests of force vs. force + corticision. Significant difference between groups is noted by $*(\mathrm{P}<0.05),(\mathrm{n}=4-5)$. 


\section{Chapter VIII: References}

1. American Association of Orthodontists 2007 How long will my treatment take? (http://www.aaomembers.org)

2. Richter AE, Arruda AO, Peters MC, Sohn W. Incidence of caries lesions among patients treated with comprehensive orthodontics. Am J Orthod Dentofacial Orthop 2011 139(5):657-64.

3. Alexander SA. Effects of orthodontic attachments on the gingival health of permanent second molars. Am J Orthod Dentofacial Orthop 1991;100:337-340.

4. Geiger AM. Mucogingival problems and the movement of mandibular incisors - a clinical review. Am J Orthod 1980; 78:511-527.

5. Weltman B, Vig KW, Fields HW, Shanker S, Kaizar EE. Root resorption associated with orthodontic tooth movement: a systematic review. Am J Orthod Dentofac Orthop 2010; 137(4): $462-76$.

6. Grunheld T, Morbach B, Zentner A. Pulpal cellular reactions to experimental tooth movement in rats. Oral Surg Oral Med Oral Pathol Oral Radiol Endod 2007;104:434-41.

7. Krishnan V, Davidovitch Z. Cellular, molecular and tissue-level reactions to orthodontic force. Am J Orthod Dentofacial Orthop 2006; 129: 469e.1-460e.32.

8. Toms SR, Lemons JE, Bartolucci AA, Eberhardt AW. Non linear stress strain behavior of periodontal ligament under orthodontic loading. Am J Orthod Dentofac Orthop 2002; 122(2): 174-9.

9. Kvinnsland, S. Effect of experimental tooth movement on periodontal and pulpal blood flow. Eur J Orthod 1989; 11(3): 200-5.

10. Davidovitch Z, Nicolay OF, Ngan PW, Shanfeld JL. Neurotransmitters, cytokines and control of alveolar bone remodeling in orthodontics. Dent Clin North Am. 1988; 32(3):411-35

11. Norevall Expression of neuropeptides during and after orthodontic tooth movement in the rate. Eur J Orthod 1995; 17(4):311-25.

12. Alhashimi, N.; Frithiof, L.; Brudvik, P.; and Bakhiet, M.: Orthodontic tooth movement and de novo synthesis of pro inflammatory cytokines Am J Orthod Dentofac Orthop 2001; 119(3):307-12.

13. Davidovitch Z. Cell biology associated with orthodontic tooth movement. In: Berkovitz BB, Moxham BJ, Newman HN, editors. The periodontal ligament in health and disease. St Louis: Mosby; 1995.

14. Harrell A Biochemical effect of mechanical stress on cultured bone cells. Calcified Tissue Research 1977; 22 Suppl: 202-7. 
15. Yamasaki K, Miura F, Suda T. Prostaglandin as a mediator of bone resorption induced by experimental tooth movement in rats. Journal of Dental Research 1980; 59(10):1635-42.

16.Meghji S, Sandy JR, Scutt AM, Harvey W, Harris M. Stimulation of bone resorption by lipoxygenase metabolites of arachidonic acid. Prostaglandins 1988; 36(2): 139-49.

17. Yamasaki K, Shibata Y, Imai S, Tani Y, Shibasaki Y, Fukuhara T. Clinical application of prostaglandin E1 upon orthodontic tooth movement. Am J Orthod 1984; 85(6):508 -518.

18. Leiker BJ, Nanda RS, Currier GF, Howes RI, Sinha PK. The effects of exogenous prostaglandins on orthodontic tooth movement in rats. Am J Orthod Dentofac Orthop 1995; 108(4): 380-8.

19. Kale S, Kocadereli I, Atilla P, Asan E. Comparison of the effects of 1, 25 dihydroxycholecalciferol and prostaglandin E2 on orthodontic tooth movement. Am J Orthod Dentofac Orthop 2004; 125(5):607-14.

20. Ashcraft MB, Southard KA, Tolley EA. The effect of corticosteroid induced osteoporosis on orthodontic tooth movement. Am J Orthod Dentofac Orthop 1992; 102(4):310-319.

21. Kalia S, Melsen B, Verna C. Tissue reaction to orthodontic tooth movement in acute and chronic corticosteroid treatement. Orthod Craniofac Res 2004; 7(1):26-34.

22. Soma S, Matsumoto S, Higuchi Y, Takano-Yamamoto T, Yamashita K, Kuriso K, et al. Local and chronic application of PTH accelerates tooth movement in rats. J Dent Res 2000; 79: 1717-24.

23.Soma S, Iwamoto M, Higuchi Y, Kuriso K. Effects of continuous infusion of PTH on experimental tooth movements in rats. J Bone Miner Res 1999; 14: 546-54.

24. Verna C, Dalstra M, Melsen B. The rate and the type of orthodontic tooth movment is influenced by bone turnover in a rat model. Eur J Orthod 2000; 22:343-52.

25. Shirazi M, Dehpour AR, Jafari F. The effect of thyroid hormone on orthodontic tooth movement in rats. J Clin Pediatr Dent 1999; 23:259-64.

26. Collins MK, Sinclair PM. The local use of vitamin D to increase the rate of orthodontic tooth movement. Am J Orthod Dentofac Orthop 1988; 94:278-284.

27. Takano-Yamamoto T, Kawakami M, Kobyashi Y, Yamashiro T, Sakuda M. The effect of local application of 1,25 dihydroxycholecalciferol on osteoclast numbers in orthodontically treated rats. J Dent Res. 1992; 71:53-59.

28. Yamaguchi M, Kasai K. Inflammation in periodontal tissues in response to mechanical forces. Arch Immunol Therap Exp 2005; 53:388-98. 
29. Krishnan V. Critical issues concerning root resorption: a contemporary review. World J Orthod 2005; 6:30-40.

30. Sekhavat, A.R., et al., Effect of misoprostol, a prostaglandin El analog, on orthodontic tooth movement in rats. Am J Orthod Dentofacial Orthop 2002; 122(5): 542-7.

31. Daskalogiannakis J, McLachlan KR. Canine retraction with rare earth magnets: an investigation of into the validity of the constant force hypothesis. Am J Orthod Dentofac Orthop 1996; 109:489-95.

32. Iwasaki L, Haack J, Nickel J, Morton J. Human tooth movement in response to continuous stress of low magnitude. Am J Orthod Dentofac Orthop 2000; 117: 175-183.

33. Proffit W, Fields H, Sarver D. The biologic basis of orthodontic therapy. Contemporary Orthodontics $4^{\text {th }}$ edition.

34. Davidovitch Z, Shanfeld JL. Cyclic AMP levels in alveolar bone of orthodontically treated cats. Arch Oral Biol 1975; 20:567-74.

35. Burstone CJ. The biophysics of bone remodeling during orthodontics - optimal force considerations. In: Norton LA, Burstone CJ, eds. The Biology of Tooth Movement. Boca Raton, Fla: CRC Press; 1989:321-334.

36. Schwarz AM. Tissue changes incident to orthodontic tooth movement. Int J Orthod 1932; 18:331-52.

37. Oppenheim A. Human tissue response to orthodontic intervention of short and long duration. Am J Orthod Oral Surg 1942; 28:263-301.

38. Reitan K. Some factors determining the evaluation of force in orthodontics. Am J Orthod 1957; 43:32-45.

39. Begg, PR. Differential force in orthodontic treatment. Am J Orthod 1956;42:481-510.

40. Andreason GF, Zwanzinger D. Experimental findings on tooth movement under two conditions of applied force. Angle Orthod 1967; 37:9-12.

41. Hixon EH, Atikian H, Callow GE, McDonald HW, Tracy RJ. Optimal force, differential force and anchorage. Am J Orthod 1969; 55: 437-456.

42. Hixon EH, Aasen TO, Arango J, Clark RA, Klasterman R, Miller SS, Odom WM. On force and tooth movement. Am J Orthod 1970; 57: 476-489.

43. Boester CH, Johnston LE. A clinical investigation of the concept of differential and optimal force in canine retraction. Angle Orthod 1974; 44: 113-9. 
44. Andreason GF, Zwanziger D. A clinical evaluation of the differential force concept as applied to the edgewise bracket. Am J Orthod 1980; 78: 25-40.

45. Stark TM, Sinclair PM. Effect of pulsed electromagnetic fields on orthodontic tooth movement. Am J Orthod Dentofac Orthop 1987; 91:91-104.

46. Darendeliler MA, Sinclair PM, Kusy RP. The effectss of samarium-cobalt magnets and pulsed electromagnetic fields on tooth movement. Am J Orthod Dentofac Orthop 1996;107:57888 .

47. Davidovitch Z, Finkelson MD, Steigman S, Shanfeld JL, Montgomery PC, Korostoff E. Electric currents, bone remodeling, and orthodontic tooth movement. I The effect of electric currents on periodontal cyclic nucleotides. Am J Orthod 1980; 77: 14-32.

48. Davidovitch Z, Finkelson MD, Steigman S, Shanfeld JL, Montgomery PC, Korostoff E. Electric currents, bone remodeling, and orthodontic tooth movement. II Increase in rate of tooth movement and periodontal cyclic nucleotide levels by combined force and electric current. Am J Orthod 1980; 77:33-47.

49.Kawasaki K, Shimizu N. Effects of low-energy laser irradiation on bone remodeling during experimental tooth movement in rats. Lasers in Surgery and Medicine 2000; 26: 282-91.

50. Tuncay OC, Killiany DM. The effect of gingival fiberotomy on the rate of tooth movement. Am J Orthod Dentofac Orthop 1986; 89:212-15.

51. Glenn RW, Weimer AD, Wentz FM, Krejci RF. The effect of gingival fiberotomy on orthodontic cuspid retraction in cats. Angle Orthod 1983; 53: 320-8.

52.Ren A, Lv T, Kang N, Zhao B, Chen Y, Bai, D. Rapid orthodontic tooth movement aided by alveolar surgery in beagles. Am J Orthod Dentofac Orthop 2007;131:160.e1-e10.

53. Baloul SS, Gerstenfeld LC, Morgan EF, Carvalho RS, Van Dyke TE, Kantarci A. Mechanism of action and morphologic changes in the alveolar bone response to selective alveolar decortication-facilitated tooth movement. Am J Orthod Dentofac Orthop 2011; 139: S83-101.

54. Murphy KG, Wilcko MT, Wilcko WM, Ferguson DJ. Periodontal accelerated osteogenic orthodontics: A description of the surgical technique. J Oral Maxillofac Surg 2009; 67:2160-66.

55.Mostafa YA, Fayed MMS, Mehanni S, ElBokle NN, Heider AM. Comparison of corticotomy-facilitated vs standard tooth movement techniques in dogs with miniscrews as anchor units. Am J Orthod Dentofac Orthop 2009; 136:570-7. 
56. Wang L, Lee W, Lei D, Liu Y, Yamashita DD, Yen SLK. Tissue response in corticotomy and osteotomy assisted tooth movements in rats: Histology and immunostaining. Am J Orthod Dentofac Orthop 2009; 136:770.e1-e11.

57. Wilcko MT, Wilcko WM, Pulver JJ, Bissada NF, Bouquot JE. Acclerated osteogenic orthodontics technique: A 1 stage surgically facilitated rapid orthodontic technique with alveolar augmentation. J Oral Maxillofac Surg 2009;67:2149-59.

58. Wilcko WM, Wilcko MT, Bouquot JE, Ferguson DJ. Rapid orthodontics with alveolar reshaping: Two case reports of decrowding. Int J Periodontics Restorative Dent 2001; 21:9-19.

59. Lino S, Sakoda S, Ito G, Nishimori T, Ikeda T, Miyawaki S. Acceleration of orthodontic tooth movement by alveolar corticotomy in the dog. Am J Orthod Dentofac Orthop 2007; 131:448.e1-e8.

60. Cohen G, Campbell PM, Rossouw PE, Buschang PH. Effects of increased surgical trauma on rates of tooth movement and apical root resorption in foxhound dogs. Orthod Craniofac Res 2010; 13:179-90.

61. Iseri H, Kisnici R, Bzizi N, Tuz H. Rapid canine retraction and orthodontic treatment with dentoalveolar distraction osteogenesis. Am J Orthod Dentofac Orthop 2005; 127:533-41.

62. Kisnici RS, Iseri H, Tuz H, Altug AT. Dentoalveolar distraction osteogenesis for rapid orthodontic canine retraction. J Oral Maxillofac Surg 2002; 60:389-94.

63. Hong AI, Qing-feng XU, Hong-fei LU, Zhi-hui MAI, Ai-qun AN, Guo-ping LIU. Rapid tooth movement through distraction osteogenesis of the periodontal ligament in dogs. Chin Med J 2008; 121(5):455-62.

64. Liou EJW, Huang CS. Rapid canine retraction throught distraction of the periodontal ligament. Am J Orthod Dentofac Orthop 1998;114:372-82.

65. Kim SJ, Park YG, Kang SG. Effects of corticision on paradental remodeling in orthodontic tooth movement. Angle Orthod 2009;79:284-91.

66. Kim SJ, Moon SU, Kang SG, Park. Effects of low level laser therapy after corticision on tooth movement and paradental remodeling. Lasers in surgery and medicine 2009;41:524-33.

67. Dibart S, Sebaoun JD, Surmenian J. Piezocision: A minimially invasive, periodontally accelerated orthodontic tooth movement procedure. Compendium Contin Educ Dent 2009; 30(6): 342-4, 346, 348-50.

68. Fitzpatrick BN. Corticotomy. Aust Dent J 1980;25:255-58. 
69. Kole H. Surgical operation on the alveolar ridge to correct occlusal abnormalities. Oral Surg Oral Med Oral Pathol Oral Radiol Endod 1959;12:515-29.

70. Duker J. Experimental animal research into segmental alveolar movement after corticotomy. J Maxillofac Surg 1975; 3:81-4.

71. Generson RM, Porter JM, Zell A, Stratigos GT. Combined surgical and orthodontic management of anterior open bite using corticotomy. J Oral Surg 1978;34:216-9.

72. Anholm M, Crites D, Hoff R. Corticotomy facilitated orthodontics. Calif Dent Assoc J 1986;7:8.

73. Gantes B, Rathbun E, Anholm M. Effects on the periodontium following corticotomy facilitated orthodontics. Case Reports. J Periodontol 1990; 61:234.

74. Suya H. Corticotomy in orthodontics, in Hosl E, Baldauf A (eds): Mechanical and Biological Basics in Orthodontic Therapy. Heidelberg, Hutlig Buch 1991, pp 207-226.

75. Frost HM. The regional acceleratory phenomenon: a review. Henry Ford Hosp Med J. 1983;31:3-9.

76. Ren Y. Maltha JC, Kuijpers-Jagtman AM. The rat as a model for orthodontic tooth movement - a critical review and a proposed solution. Eur J Orthod. 2004; 26:483-490.

77. Sato T, Iida J, Kurihara S. A histological study of the periodontal tissue changes during molar depression in rats. J Jpn Orthod Soc. 1984; 43:361-372.

78. Isaacson RJ, Lindauer SJ, Davidovitch M. On tooth movement. Angle Orthod 1993;63:305309.

79.Reitan K, Kvam E. Comparative behavior of human and animal tissue during experimental tooth movement. Angle Orthod 1971;41:1-14.

80. King GJ, Keeling SD, McCoy EA, Ward TH. Measuring dental drift and orthodontic tooth movement in response to various intial forces in adult rats. Am J Orthod Dentofac Orthop 1991; 99:456-65.

81. Nixon CE, Saviano JA, King GJ, Keeling SD. Histomorphometric study of dental pulp during orthodontic tooth movement. Journal of Endodontics 1993; 19:13-16.

82. Keeling SD, King GJ, McCoy EA, Valdez MV. Serum and alveolar bone phosphatase changes reflect bone turnover during orthodontic tooth movement. Am J Orthod Dentofac Orthop 1993; 103: 320-6. 
83. Yee JA, Turk T, Elekdag Turk S, Cheng L, Darendeliler MA. Rate of tooth movement under heavy and light continuous orthodontic forces. Am J Orthod Dentofacial Orthop 2009; 136: 150.e-150.e9

84. Owman-Moll P, Kurol J, Lundgren D. The effects of a four fold increased orthodontic force magnitude on tooth movement and root resorptions. An intra individual study in adolescents. Eur J Orthod 1996; 18: 287-294.

85. Fortin JM Translation of premolars in the dog by controlling the moment to force ration on the crown. American Journal of orthodontics 1971; 59: 541-551.

86. Gonzales C, Hotokezaka H, Yoshimatsu M, Yozgatian JH, Darendeliler MA, Yoshida N. Force magnitude and duration effects on amount of tooth movement and root resorption in the rat molar. Angle Orthodontist 2008; 78(3): 502-509.

87. Pilon J, Kuijpers-Jagtman AM, Maltha JC. Magnitude of orthodontic forces and rate of bodily tooth movement. An experimental study. Am J Orthod Dentofac Orthop 1996; 110: 1623.

88. Melsen B. Biologic reaction of alveolar bone to orthodontic tooth movement. Angle Orthodontist 1999;69(2):151-159.

89. Kilic N, Oktay H, Ersoz M. Effects of force magnitude on tooth movement: an experimental study in rabbits. Eur J Orthod 2010; 32: 154-158.

90. van Leeuwen EJ, Kuijper-Jagtman AM, Von den Hoff JW, Wagener FADTG, Maltha JC. Rate of orthodontic tooth movement after changing the force magnitude: an experimental study in beagle dogs. Orthod Craniofac Res 2010; 13:238-245.

91. Owman-Moll P, Kurol J, Lundgren D. Effects of a doubled force magnitude on tooth movement and root resorptions. An inter individual study in adolescents. Eur J Orthod 1996; 18: 141-150.

92. Burstone CJ, Groves MH. Threshold and optimum force values for maxillary anterior tooth movement. J Dent Res 1960; 39: 653.

93. Quinn RS, Yoshikawa DK. A reassessment of force magnitude in orthodontics. Am J Orthod 1985; 88(3): 252-260.

94. Sebaoun JD, Kantarci A, Turner JW, Carvalho RS, Van Dyke TE, Ferguson DJ. Modeling of trabecular bone and lamina dura following selective alveolar decortication in rats. J Periodontol 2008 79:1679-8

95. Baloul SS, Gerstenfeld LC, Morgan EF, Carvalho RS, Van Dyke TE, Kantarci A. Mechanism of action and morphologic changes in the alveolar bone response to selective 
alveolar decortication-facilitated tooth movement. Am J Orthod Dentofac Orthop 2011; 139: S83-101.

96. Dunn MD, Park CH, Kostenuik PJ, Kapila S, Giannobile WV. Local delivery of osteoprotegerin inhibits mechanically mediated bone modeling in orthodontic tooth movement. Bone 2007; 41:446-455. 